\title{
Hydrodynamical Limit for a Hamiltonian System with Weak Noise
}

\author{
S. Olla ${ }^{\star}$, S. R.S. Varadhan ${ }^{\star}$, and H. T. Yau ${ }^{\star \star \star}$ \\ Courant Institute of Mathematical Sciences, New York University, 251 Mercer Street, New York, \\ NY 10012, USA
}

Received July 24, 1992; in revised form November 16, 1992

\begin{abstract}
Starting from a general Hamiltonian system with superstable pairwise potential, we construct a stochastic dynamics by adding a noise term which exchanges the momenta of nearby particles. We prove that, in the scaling limit, the time conserved quantities, energy, momenta and density, satisfy the Euler equation of conservation laws up to a fixed time $t$ provided that the Euler equation has a smooth solution with a given initial data up to time $t$. The strength of the noise term is chosen to be very small (but nonvanishing) so that it disappears in the scaling limit.
\end{abstract}

\section{Introduction}

Let us begin by considering $N$ particles on $R^{3}$, evolving according to a system of Hamiltonian equations. If $\left(x_{\alpha}, p_{\alpha}\right), \alpha=1,2, \ldots, N$ are respectively the positions and momenta of the $N$ indivdual particles with components $\left\{x_{\alpha}^{i}, p_{\alpha}^{i}\right\}, i=1,2,3$ then the Hamiltonian equations of motion in the phase space $\left(R^{3} \times R^{3}\right)^{N}$ are given by

$$
\left\{\begin{array}{l}
\frac{d x_{\alpha}^{i}}{d t}=\frac{\partial \mathscr{H}}{\partial p_{\alpha}^{i}} \\
\frac{d p_{\alpha}^{i}}{d t}=-\frac{\partial \mathscr{H}}{\partial x_{\alpha}^{i}}
\end{array} .\right.
$$

Here $\mathscr{H}(x, p)$ is the Hamiltonian

$$
\mathscr{H}(x, p)=\frac{1}{2} \sum_{\alpha} \sum_{i=1}^{3}\left(p_{\alpha}^{i}\right)^{2}+\frac{1}{2} \sum_{\alpha \neq \beta} V\left(x_{\alpha}-x_{\beta}\right) .
$$

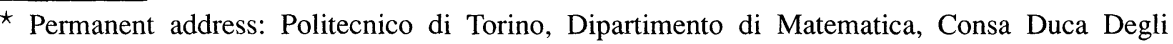
Abruzzi 24, I-10129 Torino, Italy

$\star \star$ Research partially supported by U.S. National Science Foundation grants DMS 89001682 , DMS 920-1222 and a grant from ARO, DAAL03-92-G-0317

$\star \star \star$ Research partially supported by U.S. National Science Foundation grants DMS-9101196, DMS9100383, and PHY-9019433-A01, Sloan Foundation Fellowship and David and Lucile Packard Foundation Fellowship
} 
Here $V(x)$ is a radial function defining the pair interaction which is assumed to be short range, i.e. $V(x)$ has compact support on $R^{3}$. In addition to the total number of particles which is clearly conserved there are four additional conserved quantities of interest. They are the three components of the total momenta and the total energy given by the Hamiltonian itself. We have of course assumed tacitly the masses are all equal to unity.

Let $\varepsilon>0$ be a small scale parameter. It enters in several places. The interaction $V(x)$ is assumed to have an effective range of order $\varepsilon$, so that $V(x)$ is taken to be of the form $V(x / \varepsilon)$. The number of particles $N$ is assumed to be of order $\varepsilon^{-3}$ so that even as $\varepsilon \rightarrow 0$ each particle typically interacts with a finite number of particles. We consider the following signed measures representing the spatial distribution of the conserved quantities, as a function of time:

$$
\begin{aligned}
\xi_{\varepsilon}^{0}(d x, t) & =\frac{1}{N} \sum_{\alpha} \delta\left(x-x_{\alpha}(t)\right) d x \\
\xi_{\varepsilon}^{i}(d x, t) & =\frac{1}{N} \sum_{\alpha} \delta\left(x-x_{\alpha}(t)\right) p_{\alpha}^{i}(t) d x, \quad i=1,2,3 \\
\xi_{\varepsilon}^{4}(d x, t) & =-\frac{1}{N} \sum_{\alpha} \delta\left(x-x_{\alpha}(t)\right) h_{\alpha}(t) d x \\
h_{\alpha}(t) & =\frac{1}{2} \sum_{i=1}^{3}\left(p_{\alpha}^{i}\right)^{2}+\frac{1}{2} \sum_{\beta \neq \alpha} V\left(x_{\alpha}-x_{\beta}\right) .
\end{aligned}
$$

Note that $\xi_{\varepsilon}^{4}$ has a negative sign and is the negative of energy instead of energy. We choose this convention for notational convenience later. We shall disregard this negative sign and use energy to refer to $\xi^{4}$ freely without further comment. As $\varepsilon \rightarrow 0$, $\xi_{\varepsilon}^{\mu}(d x, t)$ is expected to have limits $\xi^{\mu}(x, t) d x$ and these spatial densities of mass, momenta and energy in the limit are expected to satisfy the system of Euler equations for our model,

$$
\left\{\begin{array}{l}
\frac{d \varrho(x)}{d t}+\sum_{j=1}^{3} \frac{\partial}{\partial x_{j}}\left[\varrho(x) \pi_{j}(x)\right]=0 \\
\frac{d}{d t}\left(\varrho(x) \pi^{i}(x)\right)+\sum_{j=1}^{3} \frac{\partial}{\partial x_{j}}\left[\varrho(x) \pi^{i}(x) \pi^{j}(x)+\delta_{i j} P\right]=0 \\
\frac{d}{d t}(\varrho(x) e(x))+\sum_{j=1}^{3} \frac{\partial}{\partial x_{j}}\left[\varrho(x) e(x) \pi^{j}(x)-\pi^{j}(x) P\right]=0
\end{array}\right.
$$

Here $\varrho$ is the density; $\pi(x)$ is the velocity per particle; $e(x)$ is the energy per particle. $P(x)$ is the pressure which is a function of $\varrho, \pi$ and $e$. This is a symmetric hyperbolic system of conservation laws.

Although one expects such a result on physical grounds there is very little in the way of rigorous proof that is available (cf. [DeM, Sp, Si]). For the special case of one dimensional hard rods with elastic collision the result is known [BDS]. This case is however unusual because there are infinitely many conserved quantities.

To get back to our general 3-dimensional model we have to modify the Hamiltonian in three ways before we can establish a result of the type described earlier.

The first modification is technical in nature and we replace the physical space $R^{3}$ by a finite cube with periodic boundaries i.e. the 3 -torus $T^{3}$ (with unit volume). The 
phase space then becomes $T^{3} \times R^{3}$ for each particle. Since $V(x / \varepsilon)$ has very small support it is easily extended as a periodic function on $T^{3}$ for all sufficiently small $\varepsilon>0$.

The second modification is needed because we cannot handle the large velocities that might arise in our problem. We do not have effective truncation techniques. This forces us to modify the kinetic energy part $\frac{1}{2} \sum\left(p^{2}\right)^{2}$ to a function $\phi(p)$ which has a bounded gradient, thereby making the velocities uniformly bounded. A good example to keep in mind is the relativistic kinetic energy

$$
\phi(p)=\left(c^{2}+\sum_{i=1}^{3}\left(p^{i}\right)^{2}\right)^{1 / 2}-c^{2} .
$$

In the classical limit as $c \rightarrow \infty, \phi(p)$ becomes the classical kinetic energy.

The final modification is intrinsically more serious. This is the addition of a small amount of noise to the Hamiltonian system. This is needed because the Hamiltonian system in infinite volume has very poor ergodicity properties. One can write a family of spatially homogeneous invariant Gibbs measures on the infinite volume configuration space of positions and momenta. These are indexed by five parameters that correspond to density, the three components of average momenta and the average energy or temperature. Excluding complications of phase transition one would like to know that any spatially homogeneous invariant probability measure for the infinite volume evolution satisfying some mild regularity conditions is a superposition of these Gibbs distributions. This fact is essentially a form of a strong ergodic theorem. Since such a result is needed, but is unavailable, we modify the dynamics with noise so that the ergodic theorem we seek is available in part due to the noise. The noise itself is added only to the velocities when two particles are close, i.e. within a distance of order $\varepsilon$. They exchange velocities randomly and continuously but in such a way as to conserve the combined momenta and energy of the pair of particles. The strength of this noisy exchange is carefully regulated. It must be strong enough to provide us with the ergodicity that we need but at the same time should be weak enough that it does not alter the final Euler equations that one wants to derive. A precise formulation of the model and a statement of the results can be found in the next section.

There is a further restriction in our derivation: even if the initial data for the Euler equations are smooth and in the one-phase region, the solution will develop shocks or may enter in the region of phase coexistence. Our derivation is valid only in the smooth regime of the Euler equations.

Our approach in this paper is based on the relative entropy method used in [Y] for diffusion scaling limits (which simplifies the previous approach of [GPV] in that context).

\section{Summary}

\subsection{The Model}

The phase space in our model is $\left(T^{3} \times R^{3}\right)^{N}$. The Hamiltonian is

$$
\mathscr{H}(x, p)=\mathscr{H}_{\varepsilon, N}(x, p)=\sum_{\alpha} \phi\left(p_{\alpha}\right)+\frac{1}{2} \sum_{\alpha} \sum_{\beta \neq \alpha} V\left(\frac{x_{\alpha}-x_{\beta}}{\varepsilon}\right) .
$$


In this paper, we shall assume that $N=\varepsilon^{-3}$ for simplicity of notation. Since $V(\cdot)$ has compact support we can assume that for all sufficiently small $\varepsilon, \mathscr{H}_{\varepsilon}(x, p)$ is well defined on our phase space. Of the functions $\phi(p)$ on $R^{3}$ defining kinetic energy and $V(x)$ on $R^{3}$ defining the potential energy we assume the following:

(i) $V \in C^{1}\left(\mathbb{R}^{3}\right)$ is radial with compact support.

(ii) $V$ can be written as $V(x)=V_{0}(x)+V_{1}(x)$ where $V_{0} \geq 0, V_{0}(0)>0$ and $V_{1}$ is positive definite.

(iii) $\phi \in C^{2}\left(\mathbb{R}^{3}\right)$, strictly convex and

$$
\left|\frac{\partial \phi}{\partial p^{j}}\right|<c^{\prime}, \quad\left|\frac{\partial^{2}}{\partial p^{i} \partial p^{j}}\right|<c^{\prime \prime} \forall p .
$$

The assumption that the potential $V$ is radial is not essential, but simplifies notations.

The Hamiltonian defines a vector field on the phase space which is the Liouville operator

$$
L_{\varepsilon}=\sum \frac{\partial \mathscr{H}_{\varepsilon}}{\partial p_{\alpha}^{i}} \frac{\partial}{\partial x_{\alpha}^{i}}-\sum \frac{\partial \mathscr{H}_{\varepsilon}}{\partial x_{\alpha}^{i}} \frac{\partial}{\partial p_{\alpha}^{i}} .
$$

If it were classical evolution then we will have just $L_{\varepsilon}$. Our perturbation of $L_{\varepsilon}$ is by a second order operator involving second derivatives in $\partial / \partial p_{\alpha}^{2}$. The precise form is not important. Let us just consider two sets of momenta $\left(p_{1}^{1}, p_{1}^{2}, p_{1}^{3}\right)$ and $\left(p_{2}^{1}, p_{2}^{2}, p_{2}^{3}\right)$ constituting $R^{3} \times R^{3}=R^{6}$. The four laws of conservation are

$$
\begin{gathered}
p_{1}^{i}+p_{2}^{i}=c_{i}, \quad i=1,2,3, \\
\phi\left(p_{1}\right)+\phi\left(p_{2}\right)=c_{4} .
\end{gathered}
$$

For each $c_{1}, c_{2}, c_{3}, c_{4}$ in a certain open set we have a hypersurface $\Gamma_{c}$ in $\mathbb{R}^{6}$ of dimension 2. Observe that the assumption (iii) implies that almost all points in $\mathbb{R}^{6}$ are smooth points of the foliation $\Gamma_{c}$.

Let $\gamma(c)$ be a strictly positive function of $c_{1}, c_{2}, c_{3}, c_{4}$ that decays fast enough at infinity (for example exponentially). Let us consider the operator

$$
\hat{L}_{1,2}=-\frac{1}{2} \gamma(c) \Delta_{c ; 1,2}
$$

where $\Delta_{c ; 1,2}$ is the Laplace-Beltrami operator on the hypersurface $\Gamma_{c}$. This is just to make a canonical choice of the noise we introduced in the dynamics. We can choose in fact a more general operator $\hat{L}_{1,2}$, as long as it is elliptic of second order acting only on tangential directions of $\Gamma_{c}$. We note that the operator $\hat{L}_{1,2}$ is clearly selfadjoint with respect to Lebesgue measure on $R^{6}$. The subscript 1,2 in $\hat{L}_{1,2}$ refers to the fact that we have used particle labels 1,2 for our momenta $p_{1}^{2}$ and $p_{2}^{i}$. Clearly there are similar operators $\hat{L}_{\alpha, \beta}$ for every pair $\alpha, \beta$ and these can all be viewed as acting on functions defined on our phase space. Our noisy generator then is of the form

$$
\begin{aligned}
& \tilde{L}_{\varepsilon}=L_{\varepsilon}+\hat{L}_{\varepsilon}, \\
& \hat{L}_{\varepsilon}=\theta(\varepsilon) \sum_{\alpha \neq \beta} \psi\left(\varepsilon, x_{\alpha}-x_{\beta}\right) \hat{L}_{\alpha, \beta},
\end{aligned}
$$

where $\psi\left(\varepsilon, x_{\alpha}-x_{\beta}\right)$ provides control of the intensity of the noise and spatial cutoff for the exchange of velocities by limiting it to particles that are close in position space. The choice of the function $\psi(\varepsilon, x)$ is made as follows. We pick a function 
$\psi(x)$ which is smooth, strictly positive for all $x$ in $R^{3}$ and decays rapidly at $\infty$. Then define

$$
\psi(\varepsilon, x)=\sum_{n} \psi\left(\frac{x-n}{\varepsilon}\right) .
$$

The summation is over all the lattice points in $\mathbb{R}^{3}$ so that $\psi(\varepsilon, x)$ is a function on $T^{3}$. This essentially turns the interaction on at all distances in the scale $\varepsilon$ but with the intensity decaying very rapidly with the distance. Finally $\theta(\varepsilon)$ controls the intensity as a function of $\varepsilon$. It will go to infinity as $\varepsilon \rightarrow 0$ but such that $\varepsilon \theta(\varepsilon) \rightarrow 0$.

\subsection{The Formal Derivation of the Euler Equation}

Let us pretend for the purpose of our derivation that no noise term is present. We will associate with each configuration $\left(x_{1}, p_{1}\right), \ldots,\left(x_{N}, p_{N}\right)$ a group of five signed measures $\xi_{\varepsilon}^{\mu}(d x), \mu=0,1,2,3,4$ on $T^{3}$ :

$$
\xi_{\varepsilon}^{\mu}(d x)=\frac{1}{N} \sum_{\alpha} \delta\left(x-x_{\alpha}\right) \zeta_{\alpha, \varepsilon}^{\mu} d x
$$

where $\zeta_{\alpha, \varepsilon}^{0}=1, \zeta_{\alpha, \varepsilon}^{i}=p_{\alpha}^{i}, i=1,2,3$ and

$$
\zeta_{\alpha, \varepsilon}^{4}=-\left\{\phi\left(p_{\alpha}\right)+\frac{1}{2} \sum_{\beta \neq \alpha} V\left(\frac{x_{\alpha}-x_{\beta}}{\varepsilon}\right)\right\} .
$$

We shall adopt the convention of using $\mu, \nu$ for the indices for conserved quantities; $i$ for the three dimensions in $\mathbb{R}^{3}$ and $\alpha, \beta$ for the particles from 1 to $N=\varepsilon^{-3}$. For any test function $J(x)$ on $T^{3}$ clearly

$$
\left\langle J, \xi_{\varepsilon}^{\mu}\right\rangle=\frac{1}{N} \sum_{\alpha} J\left(x_{\alpha}\right) \zeta_{\alpha, \varepsilon}^{\mu}
$$

Because of the evolution, $\left(x_{\alpha}, p_{\alpha}\right)$ are functions $\left\{x_{\alpha}(t), p_{\alpha}(t)\right\}$ of $t$, and this makes $\xi_{\varepsilon}^{\mu}(d x)$ depend on $t$ as well. We denote these by $\xi_{\varepsilon}^{\mu}(d x, t)$. We can use the Hamiltonian equations to compute $d\left\langle J, \xi_{\varepsilon}^{\mu}(t)\right\rangle$ and write it in the following form:

$$
d\left\langle J, \xi_{\varepsilon}^{\mu}(t)\right\rangle=\sum_{\imath=1}^{3}\left\langle J_{i}, \eta_{\imath, \varepsilon}^{\mu}(t)\right\rangle d t+\Omega_{\varepsilon}^{\mu}(J) .
$$

Here

$$
\eta_{i, \varepsilon}^{\mu}(t)=\frac{1}{N} \sum_{\alpha} \delta\left(x-x_{\alpha}(t)\right) \theta_{i, \alpha, \varepsilon}^{\mu}(t)
$$


with $\theta_{i, \alpha, \varepsilon}^{\mu}$ given by

$$
\begin{aligned}
\theta_{i, \alpha, \varepsilon}^{0}(t)= & \phi_{i}\left(p_{\alpha}(t)\right) \\
\theta_{i, \alpha, \varepsilon}^{j}(t)= & p_{\alpha}^{j}(t) \phi_{i}\left(p_{\alpha}(t)\right) \\
& -\frac{1}{2} \sum_{\beta \neq \alpha} \varepsilon^{-1}\left(x_{\alpha}^{i}-x_{\beta}^{i}\right) V_{j}\left(\left(x_{\alpha}(t)-x_{\beta}(t)\right) \varepsilon^{-1}\right), \\
\theta_{i, \alpha, \varepsilon}^{4}(t)= & {\left[\zeta_{\alpha}^{4}(t) \phi_{i}\left(p_{\alpha}(t)\right)+\frac{1}{2} \sum_{\beta \neq \alpha} \varepsilon^{-1}\left(x_{\alpha}^{i}(t)-x_{\beta}^{i}(t)\right)\right.} \\
& \times\left\{\sum_{j=1}^{3} V_{j}\left(\varepsilon^{-1}\left(x_{\alpha}(t)-x_{\beta}(t)\right) \frac{1}{2}\left(\phi_{j}\left(p_{\alpha}(t)\right)+\phi_{j}\left(p_{\beta}(t)\right)\right\}\right] .\right.
\end{aligned}
$$

We have used the notation $\phi_{j}=\partial \phi / \partial p^{j}$ and $V_{j}=\partial V / \partial x_{j}$.

The $\Omega_{\varepsilon}^{\mu}$ terms are error terms and they are explicitly given by

$$
\begin{aligned}
\Omega_{\varepsilon}^{0}= & 0 \\
\Omega_{\varepsilon}^{i}= & -\frac{\varepsilon^{2}}{2} \sum_{\alpha \neq \beta}\left[J\left(x_{\alpha}(t)\right)-J\left(x_{\beta}(t)\right)-\sum_{r=1}^{3}\left(x_{\alpha}^{r}(t)-x_{\beta}^{r}(t)\right) J_{r}\left(x_{\alpha}(t)\right)\right] \\
& \times V_{\imath}\left(\frac{1}{\varepsilon}\left(x_{\alpha}(t)-x_{\beta}(t)\right)\right) \\
\Omega_{\varepsilon}^{4}= & \frac{\varepsilon^{2}}{2} \sum_{\alpha \neq \beta}\left[J\left(x_{\alpha}(t)\right)-J\left(x_{\beta}(t)\right)-\sum_{r=1}^{3}\left(x_{\alpha}^{r}(t)-x_{\beta}^{r}(t)\right) J_{r}\left(x_{\alpha}(t)\right)\right] \\
& \times\left[\sum_{s=1}^{3} V_{s}\left(\frac{1}{\varepsilon}\left(x_{\alpha}(t)-x_{\beta}(t)\right)\right) \frac{\phi_{s}\left(p_{\alpha}(t)\right)+\phi_{s}\left(p_{\beta}(t)\right)}{2}\right] .
\end{aligned}
$$

We will show that as $\varepsilon \rightarrow 0$ the $\Omega_{\varepsilon}^{\mu}$ terms become negligible.

In order to close the equations specified by (2.11) we need to express the quantities $\eta_{i, \varepsilon}^{\mu}$ as $\varepsilon \rightarrow 0$ back in terms of $\xi_{\varepsilon}^{\mu}$. This is done using Gibbs distributions (cf. Sect. 5). Given the parameters of density, the three components of momenta and average energy one expects to have a Gibbs distribution uniquely specified by these parameters. The Gibbs distribution describes the statistics of local configurations as functions of these five parameters. If one takes averages with respect to the Gibbs distribution then $\eta_{i, \varepsilon}^{\mu}$ can be replaced by $A_{i}^{\mu}(q)$ as $\xi_{\varepsilon}^{\mu}$ gets replaced by $q^{\mu}$ in the weak limit as $\varepsilon \rightarrow 0$. The Euler equations then take the form

$$
\frac{\partial q^{\mu}}{\partial t}+\sum_{r=1}^{3}\left[A_{\imath}^{\mu}(q)\right]=0, \quad \mu=0,1,2,3,4 .
$$

The matrix $A$ can be computed explicitly. If $\phi(p)=p^{2}$, then by comparing with (1.3) $A$ is given by

$$
\begin{aligned}
A_{j}^{0} & =q^{\jmath} \\
A_{\jmath}^{i} & =\delta_{i \jmath} P+q_{i} q_{j} / q_{0}, \\
A_{j}^{4} & =q^{j}\left(q_{4}-P\right) / q_{0} .
\end{aligned}
$$


Note that in order to establish a connection with the Euler equation in (1.3), $q^{\mu}$ is related to density, momenta and energy by

$$
q^{0}=\varrho, \quad q^{i}=\varrho \pi^{i} ; \quad q^{4}=\varrho e,
$$

in other words $q^{1}, q^{2}, q^{3}$, and $q^{4}$ are momenta and energy per volume instead of per particle as in the usual Euler equation (1.3). In the general case $A$ is still explicitly computable, as seen in the next section.

\subsection{A Precise Statement of the Results}

We start with our Hamiltonian in the microscopic scale on configurations of $N$ points in the phase space $\left(\Lambda \times R^{3}\right)^{N}$ where $\Lambda$ is some domain in the physical space $R^{3}$. If we denote the configuration by $\left(x_{\alpha}, p_{\alpha}\right)$ then the Hamiltonian is

$$
\mathscr{H}_{N}(p, x)=\sum_{\alpha} \phi\left(p_{\alpha}\right)+\frac{1}{2} \sum_{\alpha \neq \beta} V\left(x_{\alpha}-x_{\beta}\right) .
$$

Taking $\Lambda$ to be a bounded cube we can define a partition function

$$
\begin{aligned}
Z_{\Lambda}\left(\lambda^{0}, \lambda^{1}, \lambda^{2}, \lambda^{3}, \lambda^{4}\right)= & \sum_{N=0}^{\infty} \frac{1}{N !} \int \ldots \int_{\left(\Lambda \times \mathbb{R}^{3}\right)^{N}} \exp \left[\sum_{\alpha=1}^{N} \sum_{\mu=0}^{4} \lambda^{\mu} \zeta_{\alpha}^{\mu}\right] \\
& \times d x_{1} \ldots d x_{N} d p_{1} \ldots d p_{N}
\end{aligned}
$$

and the pressure (note the definition of pressure where the prefactor $T=\frac{1}{\lambda^{4}}$ has been omitted)

$$
\psi\left(\lambda^{0}, \ldots, \lambda^{4}\right)=\lim _{\Lambda \Uparrow R^{3}} \frac{1}{|\Lambda|} \log Z_{\Lambda} .
$$

Here $\zeta_{\alpha}^{\mu}=\zeta_{\alpha, \varepsilon=1}^{\mu}$ with $\zeta_{\alpha, \varepsilon}^{\mu}$ defined in (2.10). See Sect. 5 or [R] for the existence of the limit (2.20).

The Gibbs measure corresponding to $\lambda^{0}, \lambda^{1}, \lambda^{2}, \lambda^{3}, \lambda^{4}$ is a point process on $R^{3} \times R^{3}$ which is invariant under space translations and has the DLR property, namely the conditional distribution of the possible configurations in a box $\Lambda$, given the configuration outside has density proportional to

$$
\frac{e^{\lambda^{0} n}}{n !} \exp \left[-\lambda^{4} \tilde{\mathscr{H}}_{n}+\sum_{i=1}^{3} \sum_{\alpha=1}^{n} \lambda^{2} p_{\alpha}^{i}\right]
$$

on $\bigcup_{n}\left(\Lambda \times \mathbb{R}^{3}\right)^{n}$, where $\tilde{\mathscr{H}}_{n}$ is the Hamiltonian of the $n$-point configuration we introduced above to which has been added the interaction energy due to the configuration outside the box. This is the term

$$
\sum_{\alpha} \sum_{y} V\left(x_{\alpha}-y\right)
$$

where $\alpha$ runs over the particles inside $\Lambda$ and $y$ runs over the locations of all particles outside $\Lambda$. The general theory of equilibrium statistical mechanics provides with a set 
$W$ of possible values of $\lambda=\left(\lambda^{0}, \lambda^{1}, \lambda^{2}, \lambda^{3}, \lambda^{4}\right)$ in $R^{5}$ such that $W$ is an open set in $R^{5}$ and for each $\lambda$ in $W$ we have a unique Gibbs measure and the Gibbs measure is ergodic (with respect to space translations). The five quantities which are averages of density, momenta and energy are given as functions of $\lambda$ and they can be computed from the free energy. As $\lambda$ varies over $W$, these averages $q=\left(q^{0}, \ldots, q^{4}\right)$ vary over a set $U$ and the correspondence between $\lambda$ and $q$ is one to one and smooth as long as $\lambda$ and $q$ are restricted respectively to $W$ and $U$. To make the correspondence between $\lambda$ and $q$ transparent, let us define the thermodynamic entropy function

$$
\hat{s}(q)=\sup _{\lambda}\left[\sum_{\mu=0}^{4} q^{\mu} \cdot \lambda^{\mu}-\psi(\lambda)\right] .
$$

Then $\lambda$ and $q$ are related by the formulae

$$
\lambda^{\mu}=\partial \hat{s} / \partial q^{\mu}, \quad q^{\mu}=\partial \psi / \partial \lambda^{\mu} .
$$

Let us denote the Gibbs measure with chemical potentials $\lambda$ by $d \mu_{\lambda}$, or $d \mu_{q}$ if one specifies the parameter $q$. The matrix $A$ can now be computed explicitly by

$$
A_{j}^{\mu}(q)=\lim _{k \rightarrow \infty}(2 k)^{-3} \int \sum_{\alpha} \theta_{j, \alpha}^{\mu} I_{k}\left(x_{\alpha}\right) d \mu_{q} .
$$

Here $\theta_{j, \alpha}^{\mu}=\theta_{j, \alpha, \varepsilon=1}^{\mu}$ and $I_{k}$ is the indicator function of $D_{k}=\left\{x \in \mathbb{R}^{3}|| x_{i} \mid \leq k\right.$, $i=1,2,3\}$. Explicitly, $A$ is given by

$$
\begin{aligned}
& A_{\jmath}^{0}=-\frac{\lambda^{j}}{\lambda^{4}} q^{0}, \\
& A_{j}^{i}=-\frac{\lambda^{\imath} q^{j}}{\lambda^{4}}-\frac{P}{\lambda^{4}} \delta_{\imath, j}, \\
& A_{j}^{4}=-\frac{\lambda^{j}}{\lambda_{4}} q^{4}+\frac{\lambda^{j}}{\lambda^{4}} P .
\end{aligned}
$$

Here $\lambda=\partial \hat{s} / \partial q(2.23)$ and $P$ is the pressure as a function of $q$. By the virial theorem for dilations we have a diect relation between $P$ and the interaction $V$ (cf. [V]):

$$
\frac{1}{2} E^{\mu_{q}}\left[\sum_{\alpha \neq \beta} V_{i}\left(x_{\alpha}-x_{\beta}\right) \chi\left(x_{\alpha}\right)\left(x_{\alpha}-x_{\beta}\right)^{j}\right]=\delta_{i, j}\left(\frac{P}{\lambda^{4}}-q^{4}\right),
$$

where $\chi$ is a non-negative function with compact support and total integral 1 .

In order to see where (2.25) come from, let us compute the expectation of $p^{j} \phi_{\imath}(p)$ with respect to the density $d \omega_{\lambda}=\exp \left[\lambda^{4} \phi(p)+\boldsymbol{\lambda} \cdot \mathbf{p}\right] /$ Normalization,

$$
\int p^{j} \phi_{\imath} d \omega_{\lambda}(p)=-q^{j} \frac{\lambda^{i}}{\lambda^{4}}-\frac{\lambda_{i j}}{\lambda^{4}}
$$

The second term in the above expression is the contribution of ideal gas to the pressure tensor. This phenomena occurs also in computing $A_{j}^{i}$ and $A_{j}^{4}$.

If we give $C^{\infty}$ initial data to our hyperbolic system, up to a finite positive time, there exists a $C^{\infty}$ solution. Then we can assume that we are given a $C^{\infty}$ solution of our hyperbolic system of conservation laws in some time interval $0 \leq t \leq T_{0}$, such that $q(t, x)$ for $t \in\left[0, T_{0}\right]$ and $x \in T^{3}$ lies in a compact set $U_{1} \subset U$ with $\operatorname{dist}\left(U_{1}, \mathbb{R}^{5} \backslash U\right) \geq 2 \delta_{1}$, for some constant $\delta_{1}$. Then the corresponding $\lambda$ is a $C^{\infty}$ 
function $\lambda(t, x)$ that lies in a compact set $W_{1} \subset W$ with $\operatorname{dist}\left(W_{1}, \mathbb{R}^{5}-W\right) \geq 2 \delta_{2}$ for some constant $\delta_{2}$.

Let us consider the following time dependent density function $\hat{g}_{N}(t)$ on the phase space of $N$ particles in $\left(T^{3} \times \mathbb{R}^{3}\right)^{N}$,

$$
\hat{g}_{N}\left(t, x_{1}, \ldots, x_{N}, p_{1}, \ldots, p_{N}\right)=\frac{1}{c_{N}(t)} \exp \left[\sum_{\alpha=1}^{N} \sum_{\mu=0}^{4} \lambda^{\mu}\left(t, x_{\alpha}\right) \zeta_{\alpha, \varepsilon}^{\mu}(\mathbf{x}, \mathbf{p})\right],
$$

where $c_{N}(t)$ is the normalizing constant.

We can take $\hat{g}_{N}\left(0, x_{1}, \ldots, x_{N}, p_{1}, \ldots, p_{N}\right)$ as the initial value at $t=0$ of the Kolmogorov or Fokker-Planck equation

$$
\frac{\partial g}{\partial t}=\tilde{L}_{\varepsilon}^{*} g,\left.\quad g\right|_{t=0}=\left.\hat{g}\right|_{t=0}
$$

and the solution $g_{N}\left(t, x_{1}, \ldots, x_{N}, p_{1}, \ldots, p_{N}\right)$ is the actual distribution at time $t$ of our noisy evolution.

Our main theorem is the following:

Theorem 2.1. Under the hypotheses listed above, for any $t \in\left[0, T_{0}\right]$,

$$
\lim _{N \rightarrow \infty} \frac{1}{N} \iint_{\left(T^{3} \times R^{3}\right)^{N}} g_{N} \log \frac{g_{N}}{\hat{g}_{N}} d x_{1} \ldots d x_{N} d p_{1} \ldots d p_{N}=0 .
$$

Remark. The above theorem implies hydrodynamic behavior. For instance it is elementary to check that under $\hat{g}_{N}$-probability $\frac{1}{N} \sum J\left(x_{\alpha}\right)$ is nearly equal to $\int J(x) q^{0}(t, x) d x$ and in fact by the theory of large deviation the probability that $\frac{1}{N} \sum J\left(x_{\alpha}\right)$ deviates significantly from $\int J(x) q^{0}(t, x) d x$ is exponentially small under $\hat{g}_{N}$ (Corollary 5.8). By entropy estimates (2.29) the probability is small under $g_{N}$ and this establishes the correct hydrodynamic behavior.

More precisely, for any two probability measures $\alpha$ and $\beta$ the well known entropy inequality (in fact, a special case of Jensen's inequality) states that for any measurable function $F$,

$$
E^{\alpha}[F] \leq \log E^{\beta}[\exp F]+H(\alpha / \beta) .
$$

Here $H(\alpha / \beta)$ denotes the relative entropy of $\alpha$ with respect to $\beta$ [indeed $H$ is defined to be the smallest constant for which (2.28) holds]. A special case of (2.28) gives

$$
E^{\alpha}(A) \leq \frac{\log 2+H(\alpha / \beta)}{\log \left(1+1 / E^{\beta}(A)\right)}
$$

Hence if $N^{-1} H(\alpha / \beta) \rightarrow 0$ as $N \rightarrow \infty$ then any set $A$ which has exponentially small probability with respect to $\beta$ will have small probability with respect to $\alpha$. In our case $\beta$ is local Gibbs state $\hat{g}_{N}$ and $A$ is the set

$$
A_{\delta}=\left\{\left|\left\langle J, \xi_{\varepsilon}^{\mu}\right\rangle-\left\langle J, q^{\mu}\right\rangle\right|>\delta\right\} .
$$

For arbitrary $\delta>0, E^{\hat{g}}\left[A_{\delta}\right] \leq \exp [-\operatorname{const}(\delta) N]$ for some const $(\delta)>0$ by large deviation theory in the appendix (see Corollary 5.8). Hence we conclude the following

Corollary 2.2. Under the assumption of Theorem 2.1,

$$
\varlimsup_{\varepsilon \rightarrow 0} E^{g_{N}}\left[A_{\delta}\right]=0 \text {. }
$$

This corollary establishes the hydrodynamical limit under the usual definition. 


\section{An Outline of the Proof}

On the phase space $\left(T^{3} \times \mathbb{R}^{3}\right)^{N}$ we have two time dependent families of probability densities defined for $0 \leq t \leq T_{0}$. One of them $\hat{g}_{N}(t, \mathbf{x}, \mathbf{p})$ is a family of local Gibbs measures constructed from a smooth solution of the Euler equations, i.e.

$$
\log \hat{g}_{N}=\sum_{\alpha=1}^{N} \sum_{\mu=0}^{4} \lambda^{\mu}\left(t, x_{\alpha}\right) \zeta_{\alpha, \varepsilon}^{\mu}-\log c_{N}(t)
$$

where $\zeta$ was defined by $(2.10)$ and $c_{N}(t)$ is the normalization constant

$$
c_{N}(t)=\int \hat{g}_{N}(t, \mathbf{x}, \mathbf{p}) d \mathbf{x} d \mathbf{p} .
$$

The other family $g_{N}(t, \mathbf{x}, \mathbf{p})$ is the actual probability density of the noisy dynamics assuming that at time $t=0$ the initial distribution is the same as $\hat{g}_{N}(t, \mathbf{x}, \mathbf{p})$ at $t=0$. In other words $g_{N}$ is the solution of the Fokker-Planck or Kolmogorov equation

$$
\frac{\partial g_{N}}{\partial t}=\tilde{L}_{\varepsilon}^{*} g_{N},\left.\quad g_{N}\right|_{t=0}=\hat{g}_{N}(0, \mathbf{x}, \mathbf{p}) .
$$

Our goal is to show that $g_{N}$ is close to $\hat{g}_{N}$ for $0 \leq t \leq T_{0}$ by establishing

$$
\lim _{N \rightarrow \infty} \frac{1}{N} \int \log \frac{g_{N}}{\hat{g}_{N}} \cdot g_{N} d \mathbf{x} d \mathbf{p}=0 \text {. }
$$

Let us define

$$
H_{N}(t)=\int \log \frac{g_{N}}{\hat{g}_{N}} g_{N} d \mathbf{x} d \mathbf{p}
$$

We know by construction that $N^{-1} H_{N}(0)=0$. The first step is to establish a differential inequality for $H_{N}(t)$.

Lemma 3.1. Let $f(t, x)$ be a solution of the forward equation of a Markov process with generator $L$, being represented as densities relative to some fixed reference measure $\mu$. Let $u(t, x)$ be any arbitrary family of densities relative to $\mu$. We shall assume enough smoothness to make our computations valid. Then for every $t>0$,

$$
\frac{\partial}{\partial t} \int \log \frac{f(t, x)}{u(t, x)} f(t, x) d \mu \leq \int\left(L^{*} u-\frac{\partial u}{\partial t}\right) \cdot \frac{1}{u} f d \mu(x) .
$$

Proof. Let us differentiate $\int \log \frac{f(t, x)}{u(t, x)} \cdot f(t, x) d \mu$ with respect to $t$. An elementary calculation yields

$$
\frac{\partial}{\partial t} \int \log \frac{f(t, x)}{u(t, x)} f(t, x) d \mu=-\int \frac{1}{u} \frac{\partial u}{\partial t} \cdot f d \mu+\int L^{*} f \log \frac{f}{u} d \mu+\int \frac{\partial f}{\partial t} d \mu .
$$

The third term $\int \frac{\partial f}{\partial t} d \mu$ integrates to zero because $\int f d \mu=1$ for all $t>0$. Integrating by parts,

$$
\begin{aligned}
\int L^{*} f \log \frac{f}{u} d \mu & =\int f L \log \frac{f}{u} d \mu \leq \int f \frac{L(f / u)}{f / u} d \mu \\
& =\int u\left(L \frac{f}{u}\right) d \mu=\int\left(L^{*} u\right) \frac{f}{u} d \mu=\int\left(\frac{L^{*} u}{u}\right) f \cdot d \mu .
\end{aligned}
$$


The inequality $L \log u \leq L u / u$ for any nonegative $u$ is a consequence of the maximum principle for $L$ that dictates that for any convex function $\phi$,

$$
L \phi(u) \geq \phi^{\prime}(u) L u .
$$

Remark. For the case we are interested in with $L$ given by (2.6) the error term in Lemma 3.1 can be computed explicitly,

$$
\begin{aligned}
\frac{\partial}{\partial t} \int g_{N} \log \frac{g_{N}}{u} d \mathbf{x} d \mathbf{p}= & \int u^{-1}\left(L_{\varepsilon}^{*} u-\frac{\partial u}{\partial t}\right) g_{N} d \mathbf{x} d \mathbf{p} \\
& +\theta(\varepsilon) \int \sum_{\alpha \neq \beta} \psi\left(\varepsilon, x_{\alpha}-x_{\beta}\right) \gamma(c) \\
& \times\left|\nabla_{c ; \alpha, \beta} h_{N}\right|^{2} h_{N}^{-1} u(t, x) d \mathbf{x} d \mathbf{p}
\end{aligned}
$$

where $h=g_{N} / u$. The last term is traditionally called the Dirichlet form. In particular if we let $u=\hat{g}_{\lambda, N}$ with $\lambda$ constant, then the first term on the right side vanishes. Let $\bar{h}=T_{0}^{-1} \int_{0}^{T_{0}} h(t) d t$ be the time average and integrate from 0 to $T_{0}$,

$$
E^{\hat{g}_{\lambda, N}}\left[\sum_{\alpha \neq \beta} \psi\left(\varepsilon, x_{\alpha}-x_{\beta}\right) \gamma(c)\left|\nabla_{c ; \alpha, \beta} h_{N}\right|^{2} h_{N}^{-1}\right] \leq \frac{H_{N}(0)}{T_{0}} .
$$

Here we have used the convexity of the Dirichlet form with respect to $h$. For a more detailed explanation of this, see [GPV].

Lemma 3.1 was first proved in [Y] for the special case of the Ginzburg-Landau model. Our proof here generalizes it to include all stochastic processes.

Returning to our problem at hand let us write

$$
\log \hat{g}_{N}(t, \mathbf{x}, \mathbf{p})=u_{N}(t, \mathbf{x}, \mathbf{p}) .
$$

We want to calculate the function

$$
\psi_{N}(t, \mathbf{x}, \mathbf{p})=\hat{g}_{N}^{-1}\left[\tilde{L}^{*} \hat{g}_{N}-\frac{\partial}{\partial t} \hat{g}_{N}\right]
$$

Lemma 3.2. Recall that $\tilde{L}_{\varepsilon}=L_{\varepsilon}+\hat{L}_{\varepsilon}$, where $\hat{L}_{\varepsilon}$ is the contribution from the noise terms and $L_{\varepsilon}$ is the pure Hamiltonian term or the Liouville operator. Then

$$
\limsup _{N \rightarrow \infty} \sup _{0 \leq t \leq T_{0}} \frac{1}{N} \int\left(\hat{L}_{\varepsilon} \hat{g}_{N}\right) \cdot \frac{1}{\hat{g}_{N}} \cdot g_{N} d \mathbf{x} d \mathbf{p}=0 .
$$

Proof. Let us note that $\hat{L}_{\varepsilon}$ has the form

$$
\hat{L}_{\varepsilon}=\theta(\varepsilon) \sum \psi\left(\varepsilon, x_{\alpha}-x_{\beta}\right) \hat{L}_{\alpha, \beta}
$$

and that $L_{\alpha, \beta}$ acts only on the momentum variables. It is easy to estimate, using the conservation properties of $\hat{L}_{\alpha \beta}$,

$$
\hat{g}_{N}^{-1} \hat{L}_{\varepsilon} \hat{g}_{N} \leq C \varepsilon \theta(\varepsilon) \sum \psi\left(\varepsilon, x_{\alpha}-x_{\beta}\right) .
$$


We therefore have only to prove

$$
\limsup _{N \rightarrow \infty} \frac{1}{N} \int \sum_{\alpha, \beta} \psi\left(\varepsilon, x_{\alpha}-x_{\beta}\right) g_{n} d \mu \leq C
$$

in order to establish the lemma, because we have assumed that $\varepsilon \theta(\varepsilon) \rightarrow 0$. This is a consequence of the fast decay of $\psi$ and Lemma 5.9.

We can now concentrate on the Liouville term $\left(L_{\varepsilon}^{*}-\frac{\partial}{\partial t}\right) u_{N}$.

Lemma 3.3. We can write

$$
\left(L_{\varepsilon}^{*}-\frac{\partial}{\partial t}\right) u_{N}=\psi_{N}^{(1)}(t, x, p)+\varepsilon_{N}(t, x, p)
$$

where

$$
\psi_{N}^{(1)}(t, x, p)=-\sum_{\mu=0}^{4} \sum_{j=0}^{3} \sum_{\alpha} \lambda_{\jmath}^{\mu}\left(t, x_{\alpha}\right) \theta_{j, \alpha, \varepsilon}^{\mu}+a_{N}(t)
$$

and

$$
\limsup _{N \rightarrow \infty} \sup _{0 \leq t \leq T_{0}} \frac{1}{N} \int \varepsilon_{N}(t, x, p) g_{N} d \mu=0 .
$$

Here $\theta_{j, \alpha, \varepsilon}^{\mu}, j=1,2,3$ was defined in (2.13), $\theta_{0, \alpha, \varepsilon}^{\mu}=\zeta_{\alpha, \varepsilon}^{\mu}$ (see (2.10)), $\lambda_{0}^{\mu}=\partial \lambda^{\mu} / \partial t$, $\lambda_{j}^{\mu}=\partial \lambda^{\mu} / \partial x_{j}$ and $a_{N}$ is given by

$$
a_{N}(t)=\frac{d}{d t}-\left[\log c_{N}(t)\right]=-\int \sum_{\mu=0}^{4} \sum_{\alpha=1}^{N} \lambda_{0}^{\mu}\left(t, x_{\alpha}\right) \zeta_{\alpha, \varepsilon}^{\mu}(\mathbf{x}, \mathbf{p}) \hat{g}_{N}(t, \mathbf{x}, \mathbf{p}) d \mathbf{x} d \mathbf{p}
$$

Proof. Proof is just by direct computation (see computation in Sect. 2.2). The error term arises because of terms of the form [cf. (2.14)]

$$
\varepsilon^{-1} \sum_{\alpha, \beta} V_{i}\left(\frac{x_{\alpha}-x_{\beta}}{\varepsilon}\right) \lambda^{i}\left(t, x_{\alpha}\right)-\sum_{\alpha, \beta} \sum_{j=1}^{3} \frac{\partial \lambda^{i}}{\partial x_{j}}\left(t, x_{\alpha}\right)\left(x_{\alpha}^{j}-x_{\beta}^{j}\right) V_{\imath}\left(\frac{x_{\alpha}-x_{\beta}}{\varepsilon}\right) .
$$

Since $V_{i}$ is antisymmetric, we can rewrite it as

$$
\varepsilon^{-1} \sum_{\alpha, \beta}\left[\lambda^{i}\left(t, x_{\alpha}\right)-\lambda^{i}\left(t, x_{\beta}\right)-\sum_{j=1}^{3} \frac{\partial \lambda^{i}}{\partial x_{j}}\left(t, x_{\alpha}\right)\left(x_{\alpha}^{j}-x_{\beta}^{j}\right)\right] V\left(\left(x_{\alpha}-x_{\beta}\right) / \varepsilon\right) .
$$

By Taylor's theorem, it is bounded by

$$
\left.(2 \varepsilon)^{-1} \sum_{\alpha, \beta} \mid V_{i}\left(x_{\alpha}-x_{\beta}\right) / \varepsilon\right) \mid \cdot O\left(\varepsilon^{2}\right) .
$$

By (3.5) and Lemma 5.9, $E^{g}\left[\left|\sum_{\alpha, \beta} V_{j}\left(\left(x_{\alpha}-x_{\beta}\right) / \varepsilon\right)\right|\right] \leq$ const $N$. Thus we have
proved Lemma 3.3. $\square$ Another easy consequence of Lemma 5.9 is 
Lemma 3.4. For some constant $C<\infty$,

$$
\int \sum_{\alpha}\left|\theta_{j, \alpha}^{\mu}\right| g_{N} d x d p \leq C N, \quad \int \sum_{\alpha}\left|\theta_{j, \alpha}^{\mu}\right| \hat{g}_{N} d x d p \leq C N .
$$

For any configuration $\left(x_{\alpha}, p_{\alpha}\right)$ on $\left(T^{3} \times R^{3}\right)^{N}$ and for any $x \in T^{3}$ we shall expand a neighborhood of $x$ by $1 / \varepsilon$ and let $y_{\alpha}=\frac{1}{\varepsilon}\left(x_{\alpha}-x\right)$. The resulting configuration can be thought of as a configuration in $R^{3}$ although it is a little fuzzy at the edges. However the configuration $\left\{y_{\alpha}\right\}$ is well-defined on compact domains in $R^{3}$ provided $\varepsilon$ is sufficiently small. Of course $\varepsilon$ has to be smaller if the compact domain is larger. The configuration so obtained will be denoted by $\omega_{\varepsilon, x}$. If $F(\omega)$ is a functional on configuration space in $R^{3}$ that depends only on particles in a compact domain, then $F\left(\omega_{x, \varepsilon}\right)$ is well defined if $\varepsilon$ is small enough. Moreover we can attach the momenta $p_{\alpha}$ to each $x_{\alpha}$ so the functional $F(\omega)$ can be a functional of configurations in the phase space.

Recall $\theta_{i, x, \varepsilon}^{\mu}$ defined in (2.13). We shall denote $\theta_{\imath, \alpha, \varepsilon=1}^{\mu}$ by $\theta_{i, \alpha}^{\mu}$. Let $\chi(x)$ be a positive smooth function on $\mathbb{R}^{3}$ with compact support and total integral 1 . Define the local functional on the configurations of phase space by

$$
\theta_{i}^{\mu}(\omega)=\sum_{\alpha} \chi\left(x_{\alpha}\right) \theta_{i, \alpha}^{\mu}(\omega)
$$

Let $I_{k}(x)$ be the indicator function of $D_{k}=\left\{x \in \mathbb{R}^{3}|| x_{i} \mid \leq k\right\}$ and let $\chi_{k}=$ $(2 k)^{-3} \chi * I_{k}$. For any local functional $F$, define $F_{k}$ to be its average over $D_{k}$,

$$
F_{k}(\omega)=(2 k)^{-3} \int I_{k}(y) F\left(\tau^{y} \omega\right) d y \text {. }
$$

Here $\tau^{y}$ is the space translation by $y$. With these conventions, $\theta_{\imath, k}^{\mu}=\left(\theta_{i}^{\mu}\right)_{k}$ is well defined.

Lemma 3.5. For any smooth function $J$ and $\mu=0, \ldots, 4, j=1,2,3$,

$$
\varlimsup_{k \rightarrow \infty} \varlimsup_{N \rightarrow \infty} E^{g}\left[N^{-1}\left|\sum_{\alpha} J\left(x_{\alpha}\right) \theta_{j, \alpha, \varepsilon}^{\mu}(\omega)-\int_{T^{3}} J(x) \theta_{\jmath, k}^{\mu}\left(\omega_{\varepsilon, x}\right) d x\right|\right]=0 .
$$

Remark. Similarly we can define $\zeta_{k}^{\mu}=\left(\zeta^{\mu}\right)_{k}$ with $\zeta_{\alpha}^{\mu}=\zeta_{\alpha, \varepsilon=1}^{\mu}$ defined in (2.10). A parallel identity holds for $\zeta$. Intuitively, $\left(\zeta^{\mu}\right)_{k}\left(\omega_{e, x}\right)$ is the local empirical density, momenta and energy.

Proof. By definition

$$
\begin{aligned}
\int_{T_{3}} J(x) \theta_{i, k}^{\mu}\left(\omega_{\varepsilon, x}\right) d x & =\sum_{\alpha} \int_{T_{3}} d x J(x) \chi_{k}\left(\varepsilon^{-1}\left(x+x_{\alpha}\right)\right) \theta_{i, \alpha, \varepsilon}^{\mu} \\
& =\frac{1}{N} \sum_{\alpha} J_{k, \varepsilon}\left(x_{\alpha}\right) \theta_{i, \alpha, \varepsilon}^{\mu} .
\end{aligned}
$$

Here $J_{k, \varepsilon}$ is defined by

$$
J_{k, \varepsilon}(z)=\int_{T_{3}} J(x) N \chi_{k}\left(\varepsilon^{-1}(x+z)\right) d x .
$$


We can bound the expectation in (3.9) by

$$
E^{g}\left[\sum_{\alpha}\left|J-J_{k, \varepsilon}\right|\left(x_{\alpha}\right)\left|\theta_{j, \alpha, \varepsilon}^{\mu}(\omega)\right|\right] \leq\left\|J-J_{k, \varepsilon}\right\|_{\infty} E^{g}\left[\sum_{\alpha}\left|\theta_{j, \alpha, \varepsilon}^{\mu}\right|\right] .
$$

By Lemma 3.4, $N^{-1} E^{g}\left[\sum_{\alpha}\left|\theta_{j, \alpha, \varepsilon}^{\mu}\right|\right]$ is bounded. Lemma 3.5 thus follows from the smoothness assumption of $J$.

Combining Lemma 3.1-3.5 we have

Lemma 3.6. Assume that $\lambda^{\mu}$ is smooth up to time T. Then

$$
\begin{aligned}
& \varlimsup_{k \rightarrow \infty} \varlimsup_{N \rightarrow \infty} \sup _{0 \leq t \leq T} N^{-1} \frac{d H_{N}(t)}{d t} \\
& +E^{g}\left[\int_{T^{3}} \sum_{\mu=0}^{4} \sum_{j=0}^{3} \lambda_{j}^{\mu}(t, x) \theta_{j, k}^{\mu}\left(\omega_{x, \varepsilon}\right) d x\right]+N^{-1} a_{N}(t) \leq 0 .
\end{aligned}
$$

Furthermore, we can compute $a_{N}$ as

$$
\lim _{N \rightarrow \infty} N^{-1} a_{N}(t)=\int \sum_{\mu=0}^{4} \lambda_{0}^{\mu}(x, t) q^{\mu}(x, t) d x .
$$

Proof. The only thing that needs to be proved is the formula for $a_{N}$. But it is a consequence of Lemma 3.3 and large deviations in Sect. 5 [cf. (5.20), (5.21)].

We now introduce some cutoffs. There are two sources of unboundedness. The first kind appears due to the unboundedness of $\phi\left(p_{\alpha}\right)$ and $p_{\alpha}^{i}$. Let us introduce a cutoff $l$ and replace $\phi\left(p_{\alpha}\right)$ by $\min \left\{\phi\left(p_{\alpha}\right), l\right\}$ (similarly for $p_{\alpha}^{i}$ ). Let us denote the resulting $\theta$ with these replacements by $\theta_{j, \alpha, \varepsilon, l}^{\mu}, \theta_{j, k, l}^{\mu}$, etc. The next lemma shows that the error we made is negligible. Its proof is again an easy application of the entropy inequality (2.28).

Lemma 3.7. For $\mu=0, \ldots, 4, j=1,2,3$,

$$
\varlimsup_{l \rightarrow \infty} \varlimsup_{N \rightarrow \infty} E^{\tilde{g}_{N}}\left[\int_{T^{3}}\left|\theta_{j, k, l}^{\mu}\left(\omega_{x, \varepsilon}\right)-\theta_{j, k}^{\mu}\left(\theta_{x, \varepsilon}\right)\right| d x\right]=0 .
$$

Here $k$ is arbitrary positive and $\tilde{g}_{N}$ can either be $g_{N}$ (3.3) or $\hat{g}_{N}$ (3.1).

The next step we have to be concerned about is that some of the empirical quantities $\zeta_{k}^{\mu}$ characterizing the local equilibrium states may not lie in the admissible region $U$ defined before (2.22). Let $U_{2}$ be a compact set satisfying [with $\delta_{2}$ defined before (2.22)]

$$
\operatorname{dist}\left(U_{1}, \mathbb{R}^{3} \backslash U_{2}\right) \geq \delta_{2}, \quad \operatorname{dist}\left(U_{2}, \mathbb{R}^{3} \backslash U\right) \geq \delta_{2} .
$$

Define an indicator function $\sigma_{k}$ by

$$
\sigma_{k}(\omega)=1_{U_{2}}\left\{\zeta_{k}^{\mu}(\omega)\right\}
$$

The next lemma states that the error we made from introducing this cutoff is again negligible. Its proof relies on large deviation and will be proved in Sect. 5 after (5.22). 
Lemma 3.8. There exist $\delta_{0}>0$ such that for any local functional $A$ of the type (5.18),

$$
\varlimsup_{k \rightarrow \infty} \varlimsup_{N \rightarrow \infty} E^{g_{N}}\left[\int_{T^{3}}\left(1-\sigma_{k}\right)\left(\omega_{\varepsilon, x}\right) A\left(\omega_{\varepsilon, x}\right) d x\right]-\delta_{0}^{-1} N^{-1} H\left(g_{N} \mid \hat{g}_{N}\right) \leq 0 .
$$

Now we state the local ergodic theorem to be proved in the next section.

Definition 3.9. For any local functional $F(\omega)$ define

$$
\hat{F}(q)=E^{\mu q}[F] .
$$

Here $\mu_{q}$ is the unique Gibbs measure with average density, momenta and energy given by $q$. This is well defined if $q \in U$.

The next theorem will be proved in Sect. 4.

Theorem 3.10 (Local ergodic theorem.) For any bounded local functional $F$ we have

$$
\varlimsup_{k \rightarrow \infty} \varlimsup_{N \rightarrow \infty} \int_{0}^{T} d t E_{N}^{g}\left[\int_{T^{3}}\left|F_{k}\left(\omega_{\varepsilon, x}\right)-\hat{F}\left(\zeta_{k}\left(\omega_{\varepsilon, x}\right)\right)\right| \sigma_{k}\left(\theta_{\varepsilon, x}\right) d x\right]=0 .
$$

Let us pause to see what we have so far. By Lemma 3.6 and cutoffs in Lemmas 3.7 and 3.8 we have

$$
\begin{aligned}
& \varlimsup_{l \rightarrow \infty} \varlimsup_{k \rightarrow \infty} \varlimsup_{N \rightarrow \infty} \sup _{0 \leq t \leq T} N^{-1} \frac{d H_{N}(t)}{d t} \\
& +E^{g}\left[\int_{T^{3}} \sum_{\mu=0}^{4} \sum_{\jmath=0}^{3} \lambda_{\jmath}^{\mu}(t, x) \theta_{\jmath, k, l}^{\mu}\left(\omega_{x, \varepsilon}\right) \sigma_{k}\left(\omega_{x, \varepsilon}\right) d x\right] \\
& +N^{-1} a_{N}(t)-\delta_{0}^{-1} N^{-1} H_{N}(t) \leq 0
\end{aligned}
$$

By Theorem 3.10 we can replace $\theta_{j, k, l}^{\mu}\left(\omega_{x, \varepsilon}\right)$ by $\hat{\theta}_{j, l}^{\mu}\left(\boldsymbol{\zeta}_{k}\left(\omega_{\varepsilon, x}\right)\right)$. By applying Lemma 3.7 to $\hat{g}_{N}$ we can replace $\hat{\theta}_{j, l}^{\mu}$ by $\hat{\theta}_{j}^{\mu}$ in the limit $l \rightarrow \infty$ and thus we can rewrite (3.11) as

$$
\begin{aligned}
& \varlimsup_{l \rightarrow \infty} \varlimsup_{k \rightarrow \infty} \varlimsup_{N \rightarrow \infty} \sup _{0 \leq t \leq T} N^{-1} \frac{d H_{N}(t)}{d t} \\
& \quad+E^{g_{N}}\left[\int_{T^{3}} \sum_{\mu=0}^{4} \sum_{\jmath=0}^{3} \lambda_{j}^{\mu}(t, x) \hat{\theta}_{j}^{\mu} \cdot I_{U_{2}}\right)\left(\zeta_{k}\left(\omega_{x, \varepsilon}\right)\right] d x \\
& \quad+N^{-1} a_{N}(t)-\delta_{0}^{-1} N^{-1} H_{N}(t) \leq 0 .
\end{aligned}
$$

The function $\hat{\theta}_{j}^{\mu}$ can be easily computed. By definition of $\theta$ in Lemma 3.3, (2.24) and (2.25),

$$
\hat{\theta}_{j}^{\mu}(q)=A_{j}^{\mu}(q), \quad j=1,2,3, \mu=0,1,2,3,4 .
$$

Clearly, by definition $\theta_{0, \alpha}^{\mu}=\zeta_{\alpha}^{\mu}$ and thus

$$
\hat{\theta}_{0}^{\mu}(q)=q^{\mu}
$$


Observe that, since $q(x)$ is smooth, integrating by parts we have by (2.25)

$$
\int \sum_{\mu=0}^{4} \sum_{j=1}^{3} A_{\jmath}^{\mu}(q(x)) \lambda_{j}^{\mu}(q(x)) d x=0 .
$$

Hence we can rewrite the expectation in (3.12) as

$$
E^{g_{N}}\left[\int_{T^{3}} \Omega\left(\lambda(t, x), \zeta_{k}\left(\omega_{\varepsilon, x}\right)\right) d x\right]
$$

where

$$
\Omega(\boldsymbol{\lambda}, \boldsymbol{\zeta})=\left\{\sum_{\mu=0}^{4} \sum_{\jmath=1}^{3} \lambda_{j}^{\mu}\left[A_{j}^{\mu}(\boldsymbol{\zeta})-A_{j}^{\mu}(\mathbf{q})\right]+\sum_{\mu=0}^{4} \lambda_{0}^{\mu}\left(\zeta^{\mu}-q^{\mu}\right)\right\} I_{U_{2}}(\boldsymbol{\zeta})
$$

with $q=\partial \psi / \partial \lambda(2.23)$. By entropy bound (2.28) for any constant $\delta>0$,

$$
E^{g_{N}}\left[\int_{T^{3}} \Omega d x\right] \geq-(\delta \cdot N)^{-1} \log E^{\hat{g}_{N}}\left[\exp \left\{-\delta \cdot N \int_{T^{3}} \Omega d x\right\}\right]-\delta^{-1} H_{N} / N
$$

By the large deviation theory in Sect. 5 (5.21) the expectation with respect to $\hat{g}_{N}$ can be computed explicitly as

$$
\begin{gathered}
\varlimsup_{k \rightarrow \infty} \varlimsup_{N \rightarrow \infty} N^{-1} \log E^{\hat{g}_{N}}\left\{\exp \left[-\delta \cdot N \int_{T^{3}} \Omega d x\right]\right\} \\
=\sup _{\zeta(x)}\left[-\delta \int \Omega(\lambda(x), \zeta(x)) d x-I(\zeta(x), \lambda(x))\right] .
\end{gathered}
$$

Here the sup is over all integrable functions $\zeta(x)$ and

$$
I(\zeta, \lambda)=\hat{s}(\zeta)+\psi(\lambda)-\lambda \zeta
$$

with $\hat{s}$ and $\psi$ defined in (2.21) and (2.20). The function $I(\zeta, \lambda)$ is uniformly strictly convex and vanishing at $\zeta=q=\partial \psi / \partial \lambda$. Also the function $\Omega(\lambda, \zeta)$ vanishes if $\zeta=q=\partial \psi / \partial \lambda$. If we have the first derivatives of $\Omega$ also vanish at $\zeta=q$, then since $\Omega$ is bounded (thanks to the cutoff $I_{U_{2}}$ ) the sup is zero. To summarize, (3.17) is zero provided

$$
\left.\frac{\partial \Omega}{\partial \zeta^{\mu}}\right|_{\zeta=0}=0, \quad \mu=0, \ldots, 4
$$

A direct computation shows that (3.18) is equivalent to

$$
\lambda_{0}^{\mu}+\sum_{\nu, j} \lambda_{\jmath}^{\nu} \frac{\partial A^{\nu}}{\partial q^{\mu}}=0 .
$$

By (2.15)

$$
\lambda_{0}^{\mu}=\sum_{\alpha} \frac{\partial \lambda^{\mu}}{\partial q^{\alpha}} \frac{\partial q^{\alpha}}{\partial t}=\sum_{\alpha} \sum_{\beta} \sum_{\jmath} \frac{\partial \lambda^{\mu}}{\partial q^{\alpha}} \frac{\partial A_{j}^{\alpha}}{\partial q^{\beta}} \frac{\partial q^{\beta}}{\partial x_{j}} .
$$


Hence (3.19) is satisfied provided the Euler equation (2.15) is satisfied and

$$
\sum_{\alpha} \frac{\partial \lambda^{\mu}}{\partial q^{\alpha}} \frac{\partial A_{j}^{\alpha}}{\partial q^{\beta}}=\sum_{\nu} \frac{\partial \lambda^{\nu}}{\partial q^{\beta}} \frac{\partial A_{j}^{\nu}}{\partial q^{\mu}}, \quad j=1,2,3 .
$$

Since $\lambda=\partial \hat{s} / \partial \zeta$, we can write (3.20) as

$$
\sum_{\alpha} \frac{\partial^{2} \hat{s}}{\partial q^{\alpha} \partial q^{\mu}} \frac{\partial A_{j}^{\alpha}}{\partial q^{\beta}}=\sum_{\nu} \frac{\partial^{2} \hat{s}}{\partial q^{\beta} \partial q^{\nu}} \frac{\partial A_{j}^{\nu}}{\partial q^{\mu}}, \quad j=1,2,3 .
$$

This means that the Euler equation is symmetric with symmetrizer $\partial^{2} \hat{s} / \partial \zeta \partial \zeta$. One can check by explicit computation that (3.21) holds and thus conclude our main Theorem 2.1. In fact consider the rate of change of local entropy and using the Euler equation we have [by (2.25)]

$$
\frac{d}{d t} \int_{T^{3}} \hat{s}(q) d x=\int_{T^{3}} \sum_{\alpha} \frac{\partial \hat{s}}{\partial q^{\alpha}} \frac{\partial A_{j}^{\alpha}}{\partial q^{\beta}} \frac{\partial q^{\beta}}{\partial x^{j}} d x=0 .
$$

Since (3.22) is zero with respect to all choices of initial conditions, (3.22) is identical to zero even if we take $q^{\alpha}$ as independent variables. Hence the compatibility conditions require

$$
\frac{\partial}{\partial q^{\beta}}\left[\frac{\partial \hat{s}}{\partial q^{\alpha}} \frac{\partial A_{j}^{\alpha}}{\partial q^{\beta}}\right]=\frac{\partial}{\partial q^{\alpha}}\left[\frac{\partial \hat{s}}{\partial q^{\beta}} \frac{\partial A_{j}^{\beta}}{\partial q^{\alpha}}\right], \quad j=1,2,3 .
$$

Doing the differentiation and using $\partial \hat{s} / \partial q^{\beta} \partial q^{\alpha}=\partial^{2} \hat{s} / \partial q^{\alpha} \partial q^{\beta}$ we also conclude (3.21).

\section{Local Ergodic Theorem}

The aim of this section is to prove Theorem 3.10. To achieve this we proceed in several steps.

Let $\varepsilon>0$ be given. Then we defined earlier (paragraph after Lemma 3.4) a local map $\omega_{\varepsilon, x}$ which maps a local configuration in the phase space around $x$ to a configuration in $\mathbb{R}^{3}$. As $\varepsilon \rightarrow 0$ the resulting configurations are getting well defined in larger and larger cubes around the origin in $\mathbb{R}^{3}$. Let us denote the measure obtained from the density $g(t, \mathbf{x}, \mathbf{p})$ through the map by $Q_{t, x, \varepsilon}$. We define

$$
\bar{Q}_{\varepsilon}=\frac{1}{T_{0}} \int_{0}^{T_{0}} d t \int_{T^{3}} d x Q_{t, x, \varepsilon}
$$

Roughly speaking, if we pick a random time " $t$ " in $\left[0, T_{0}\right]$ and a random point " $x$ " in $T^{3}$ and blow up the space by a factor of $\varepsilon^{-1}$, the statistics of the configuration in phase space in a large $\varepsilon$-vicinity of this point is given by $\bar{Q}_{\varepsilon}$. As $\varepsilon \rightarrow 0, \bar{Q}_{\varepsilon}$ gets to be well defined as a point process on all of $\mathbb{R}^{3}$ :

The following list contains the main steps in proving local ergodic theorem (3.10). (A) The family $\left\{\bar{Q}_{\varepsilon}\right\}$ is compact. It also follows that any limiting point process has finite entropy. 
(B) Conditioned on the positions $\mathbf{x}$, the distribution of the momenta is a mixture of product states $\prod_{i} \exp \left\{-a \phi\left(p_{i}\right)-b p_{i}\right\}$ /Normalization. This can be proven with the help of the noise introduced in the dynamics.

(C) We shall define the energy, momenta and density associated with a configuration and prove that they are constants of the motion determined by the generator $L$ (in the sense specified later).

(D) By conditioning on energy, momenta and density, any limiting process is invariant under the Liouville operator $L$ and hence is Gibbs with potential $V$.

(E) The local ergodic Theorem 3.10 is just the law of large numbers for Gibbs states.

Lemma 4.1. As $\varepsilon \rightarrow 0$ the family $\left\{\bar{Q}_{\varepsilon}\right\}$ of point processes is compact and any limit point is a stationary point process in $\mathbb{R}^{3}$ with values in momentum space.

Proof. To prove compactness we have to prove that:

$$
\begin{gathered}
E^{\bar{Q}_{\varepsilon}}\left[\sum_{\alpha} \mathbf{1}_{\Lambda}\left(x_{\alpha}\right)\right] \leq|\Lambda| \\
\lim _{l \rightarrow \infty} \limsup _{\varepsilon \rightarrow 0} E^{\bar{Q}_{\varepsilon}}\left[\sum_{\alpha} \mathbf{1}_{\Lambda}\left(x_{\alpha}\right)\left(1-\mathbf{1}_{l}\left(p_{\alpha}\right)\right)\right]=0
\end{gathered}
$$

for any bounded set $\Lambda$ in $\mathbb{R}^{3}$. Here $\mathbf{1}_{l}(p)$ is the characteristic function of the set $\{p:\|p\| \leq l\}$. In (4.2) actually equality holds. In fact an elementary computation yields:

$$
E^{\bar{Q}_{\varepsilon}}\left[\sum_{\alpha} \mathbf{1}_{\Lambda}\left(x_{\alpha}\right)\right]=\frac{1}{T_{0}} \int_{0}^{T_{0}} d t \int_{T^{3}} d x \sum_{\alpha} \mathbf{1}_{\Lambda}\left(\varepsilon^{-1}\left(x_{\alpha}-x\right)\right) g(t, \mathbf{x}, \mathbf{p}) d \mathbf{x} d \mathbf{p}
$$

Since

$$
\int_{T^{3}} \mathbf{1}_{\Lambda}\left(\varepsilon^{-1}\left(x_{\alpha}-x\right)\right) d x=\varepsilon^{3}|\Lambda|=N^{-1}|\Lambda|
$$

(4.2) follows.

Let us denote by $\bar{g}_{N}(\mathbf{x}, \mathbf{p})=\frac{1}{T_{0}} \int_{0}^{T_{0}} g(t, \mathbf{x}, \mathbf{p}) d t$. Then

$$
\begin{aligned}
E^{\bar{Q}_{\varepsilon}} & \left(\sum_{\alpha} \mathbf{1}_{\Lambda}\left(x_{\alpha}\right)\left(1-\mathbf{1}_{l}\left(p_{\alpha}\right)\right)\right) \\
= & \iint_{T^{3}} d x \sum_{\alpha} \mathbf{1}_{\Lambda}\left(\varepsilon^{-1}\left(x_{k}-x\right)\right)\left(1-\mathbf{1}_{l}\left(p_{\alpha}\right)\right) \bar{g}_{N}(\mathbf{x}, \mathbf{p}) d \mathbf{x} d \mathbf{p} \\
& =|\Lambda| \varepsilon^{3} \int \sum_{\alpha}\left(1-\mathbf{1}_{l}\left(p_{\alpha}\right)\right) \bar{g}_{N}(\mathbf{x}, \mathbf{p}) d \mathbf{x} d \mathbf{p} .
\end{aligned}
$$


We can use as a reference measure a Gibbs measure $\hat{g}_{\lambda, N}=\exp \left\{\sum_{\alpha=1} \sum_{\mu=0}^{4} \lambda^{\mu} \zeta_{\alpha, \varepsilon}^{\mu}+\right.$ $\left.c_{N, \lambda}\right\}$. Then by the entropy bound (2.28):

$$
\begin{aligned}
\frac{|\Lambda|}{N} \int \sum_{\alpha}\left[1-\mathbf{1}_{l}\left(p_{\alpha}\right)\right] \bar{g}_{N}(\mathbf{x}, \mathbf{p}) d \mathbf{x} d \mathbf{p} \leq & \frac{|\Lambda|}{\delta N}\left\{\log \int e^{\delta \sum_{\alpha}\left(1-\mathbf{1}_{l}\left(p_{\alpha}\right)\right)} \bar{g}_{\lambda, N}(\mathbf{x}, \mathbf{p}) d \mathbf{x} d \mathbf{p}\right\} \\
& +\frac{|\Lambda|}{\delta N} H_{N}\left(\bar{g}_{N} \mid \hat{g}_{\lambda, N}\right) .
\end{aligned}
$$

Since $H_{N}(g(0, \mathbf{x}, \mathbf{p}) \mid \hat{g} \lambda) \leq C N$ for some $C>0$, and decreases in time, by convexity we have

$$
H_{N}\left(\bar{g}_{N} \mid \hat{g}_{\lambda, \mu}\right) \leq \frac{C N}{T_{0}}
$$

We let $N \rightarrow \infty$ and $l \rightarrow \infty$. Note that $\left\{p_{\alpha}\right\}_{\alpha=1}^{N}$ are independent under $\hat{g}_{\lambda}$ and the first term can be calculated explicitly and goes to zero as $l \rightarrow \infty$. Finally let $\delta \rightarrow \infty$ and (4.3) follows.

Lemma 4.2 Let $Q$ be any limit point of $\left\{\bar{Q}_{\varepsilon}\right\}$. For any $\delta>0$ let $P_{\delta}$ the Poisson process on $\mathbb{R}^{3} \times \mathbb{R}^{3}$ with intensity $d x \times \frac{e^{-\delta \phi(p)}}{c_{\delta}} d p$ (cf. Sect. 5 , $c_{\delta}$ is a normalization constant). Then there exists a constant $C<\infty$ independent of $Q$ such that the density of relative entropy

$$
h\left(Q \mid P_{\delta}\right) \leq C
$$

( $h$ is defined by 5.1 ).

The proof of Lemma 4.2 is a simple consequence of 4.4 and the results contained in Sect. 5. We will postpone the proof to the end of Sect. 5.

Lemma 4.3. Let $Q$ be any limit point. Then for any bounded function $W$ with compact support contained in $D_{r}=\{|x| \leq r\}$ we have

$$
E^{Q}\left(\sum_{\alpha \neq \beta} W\left(x_{\alpha}-x_{\beta}\right) I_{D_{r}}\left(x_{\alpha}\right)\right) \leq \text { const } r^{6} \text {. }
$$

In particular, let $N_{r}=\sum_{\alpha} I_{D_{r}}\left(x_{\alpha}\right)$. Then

$$
E^{Q}\left(N_{r}^{2}\right) \leq \text { const } r^{6}
$$

Lemma 4.3 is the "local version" of Lemma 5.9 and can be proved in the same way.

Lemma 4.4. If $Q$ is any limit point and we restrict $Q$ to any region $W$ then $Q$ is a measure on the union of particle spaces $\Omega_{n} . \Omega_{n}$ consists of $n$ copies of the phase-space over $W$, i.e. $\left(W \times R^{3}\right)^{n}$. The restriction of $Q$ of $\Omega_{n}$ is invariant with respect to $\hat{L}_{\alpha, \beta}$ (2.6) for every $\alpha, \beta \in\{1,2, \ldots, n\}$ provided $n \geq 2$.

Proof. We will use here the assumption made on the random interaction, i.e.

$$
\psi(\varepsilon, x)>\delta_{k} \quad \text { if }\left|\varepsilon^{-1} x\right|<k .
$$

Using again $\hat{g}_{\lambda, N}$ as reference measure (for any $\lambda \in V$ ), we have from (3.5),

$$
E^{\hat{g}_{\lambda, N}}\left[\theta(\varepsilon) \sum_{\alpha \neq \beta} \psi\left(\varepsilon, x_{\alpha}-x_{\beta}\right) \gamma(c) \frac{\left|\nabla_{c ; \alpha, \beta} \bar{g}_{N}\right|^{2}}{\bar{g}_{N}}\right] \leq c^{\prime} N
$$


Since $\theta(\varepsilon) \rightarrow \infty$ as $\varepsilon \rightarrow 0$ and $\psi$ is uniformly positive in the sense described above, the assertion follows by elementary considerations by taking the limit as $N \rightarrow \infty$.

Lemma 4.5. Let $Q$ be any limit point of $\left\{\bar{Q}_{\varepsilon}\right\}$. Then $Q$, when restricted to $\Omega_{n}$ for $n \geq 2$ and given the configuration of positions, is uniform on every energy-momentum hypersurface

$$
\begin{gathered}
\sum_{\alpha=1}^{n} \phi\left(p_{\alpha}\right)=C_{0}, \\
\sum_{\alpha=1}^{n} p_{\alpha}^{i}=C_{i}
\end{gathered}
$$

(uniform in the sense of the restriction of the volume element $d p$ ).

Proof. This requires that $\sum_{\alpha, \beta} \hat{L}_{\alpha, \beta}$ be elliptic on the above hypersurface and that the hypersurface be connected. Our assumptions on $\phi$ ensure this except for special values of $c_{0}, c_{1}, c_{2}, c_{3}$ when the hypersurface collapses to a point.

Lemma 4.6. Given the configuration of positions then the configuration of momenta is a mixture of product measures of the form

$$
\pi(d p \mid \lambda)=\exp \left[\sum_{i=1}^{3} \sum_{\alpha} \lambda^{i} p_{\alpha}^{i}-\lambda^{4} \sum_{\alpha} \phi\left(p_{\alpha}\right)\right] / \text { Normalization },
$$

where $\lambda^{i}$ are constants restricted to the region where the exponent becomes negative as $\left|p_{\alpha}\right| \rightarrow \infty$.

Proof. Lemma 4.6 is just a consequence of Lemma 4.5 and the equivalence of ensembles theorem.

In the following $L$ will denote the Liouville operator defined on $\Omega$, the space of configurations in the infinite volume.

Lemma 4.7. If $Q$ is any limit point of $\left\{\bar{Q}_{\varepsilon}\right\}$ then

$$
\int L F(\omega) d Q(\omega)=0
$$

for all bounded smooth local functionals $F$.

Proof. By definition we can choose a subsequence such that for any bounded smooth local function $F$

$$
E^{Q}[L F]=\lim _{\varepsilon \rightarrow 0} E^{\bar{Q}_{\varepsilon}}[L F] .
$$

It is therefore enough to prove that $E^{\bar{Q}_{\varepsilon}}[L F] \rightarrow 0$ as $\varepsilon \rightarrow 0$. Define $G(\omega)$ by

$$
G(\omega)=(L F)(\omega)=\sum_{\alpha} \sum_{i=1}^{3} \phi_{i}\left(p_{\alpha}\right) \frac{\partial F}{\partial x_{\alpha}^{i}}-\sum_{\alpha \neq \beta} \sum_{i=1}^{3} V_{i}\left(x_{\alpha}-x_{\beta}\right) \frac{\partial F}{\partial p_{\alpha}^{2}} .
$$

Define $\bar{g}_{N}$ to be the average of $g_{N}$

$$
\bar{g}_{N}(\mathbf{x}, \mathbf{p})=\frac{1}{T} \int_{0}^{T} g_{N}(t, \mathbf{x}, \mathbf{p}) d t
$$


Then we can express $E^{\bar{Q}_{\varepsilon}}[G(\omega)]$ by

$$
E^{\bar{Q}}[G(\omega)]=E^{\bar{g}}\left\{\int_{T^{3}} G\left(\omega_{\varepsilon, x}\right) d x\right\}
$$

Define $\bar{F}_{\varepsilon}$ to be the space average of $F\left(\omega_{\varepsilon, x}\right)$,

$$
\bar{F}_{\varepsilon}(\mathbf{x}, \mathbf{p})=\int_{T^{3}} F\left(\omega_{\varepsilon, x}\right) d x
$$

Recall $L_{\varepsilon}(2.4)$ is the Liouville operator with scale $\varepsilon$. We can compute $L_{\varepsilon} \bar{F}_{\varepsilon}$ as

$$
L_{\varepsilon} \bar{F}_{\varepsilon}=\varepsilon^{-1} \int_{T^{3}} G\left(\omega_{\varepsilon, x}\right) d x .
$$

Recall by Ito's formula

$$
\begin{aligned}
E^{g_{N}} & {\left[\int_{0}^{T} \varepsilon \tilde{L}_{\varepsilon} \bar{F}_{\varepsilon}(\mathbf{x}(t), \mathbf{p}(t)) d t\right] } \\
& =\varepsilon E^{g_{N}}\left[\bar{F}_{\varepsilon}(\mathbf{x}(T), \mathbf{p}(T))\right]-\varepsilon E^{g_{N}}\left[\bar{F}_{\varepsilon}(\mathbf{x}(0), \mathbf{p}(0))\right] .
\end{aligned}
$$

Since $\bar{F}_{\varepsilon}$ is bounded, the right side of (4.11) vanishes as $\varepsilon \rightarrow 0$. By definition of $\tilde{L}_{\varepsilon}$ and (4.10) we have

$$
\lim _{\varepsilon \rightarrow 0} E^{\bar{Q}_{\varepsilon}}[G(\omega)]=\lim _{\varepsilon \rightarrow 0} E^{\bar{g}_{N}}\left[\varepsilon \hat{L}_{\varepsilon} \bar{F}_{\varepsilon}(\mathbf{x}, \mathbf{p})\right] .
$$

By assumption that $F$ is bounded smooth function we can bound $\hat{L}_{\varepsilon} \bar{F}_{\varepsilon}$ by

$$
\left|E^{\hat{g}_{N}}\left[\hat{L}_{\varepsilon} \bar{F}_{\varepsilon}(\mathbf{x}, \mathbf{p})\right]\right| \leq \varepsilon^{4} \text { const } \theta(\varepsilon) E^{\bar{g}_{N}}\left[\sum_{\alpha \neq \beta} \psi\left(\varepsilon, x_{\alpha}-x_{\beta}\right)\right] .
$$

By Lemma 5.9 and the fast decay of $\psi, \varepsilon^{3} E^{\bar{g}_{N}}\left[\sum_{\alpha \neq \beta} \psi\left(\varepsilon, x_{\alpha}-x_{\beta}\right)\right] \leq$ const. Since $\varepsilon \theta(\varepsilon) \rightarrow 0, \lim _{\varepsilon \rightarrow 0} E^{\bar{Q} \varepsilon}[G(\omega)]=0$. This proves Lemma 4.7.

Lemma 4.8. For any configuration $\omega=\left\{\left(x_{\alpha}, p_{\alpha}\right)\right\}$, let $\mathbf{z}$ be the density, momenta and kinetic energy associated with the configuration defined by

$$
\begin{aligned}
& z^{\mu}(\omega)=\lim _{\delta \rightarrow 0} z_{\chi, \delta}^{\mu}(\omega)=\lim _{\delta \rightarrow 0} \delta^{3} \sum_{\alpha=1}^{N} \chi\left(\delta x_{\alpha}\right) \zeta_{\alpha}^{\mu}(\omega), \quad \mu=0,1,2,3 \\
& z^{4}(\omega)=\lim _{\delta \rightarrow 0} z_{\chi, \delta}^{\mu}(\omega)=\lim _{\delta \rightarrow 0} \delta^{3} \sum_{\alpha=1}^{N} \chi\left(\delta x_{\alpha}\right) \phi\left(p_{\alpha}\right) .
\end{aligned}
$$

Here $\zeta_{\alpha}^{\mu}$ was defined in (2.10) and $\chi$ is a cutoff function of total integral one (cf. (3.7)). For any limit point $Q$ of $\left\{\bar{Q}_{\varepsilon}\right\}, \mathbf{z}^{\mu}(\omega)$ exist almost everywhere and are independent of the cutoff $\chi$. Furthermore, $\mathbf{z}(\omega)$ are constants of the motion defined by $L$ in the sense that

$$
\int h(\mathbf{z}(\omega)) L F(\omega) d Q=0
$$


for all local smooth functionals $F$ and all smooth functions $h$ with compact support.

Proof. Because of the stationarity of $\left\{x_{\alpha}\right\}$ as a point process and the conditionals of $p$ being independent (4.8), these limits clearly exist if the moments are finite: essentially one needs finiteness of the kinetic energy density or

$$
\int z^{4}(\omega) d Q<\infty
$$

This is implied by (4.5) and (2.28). It also follows from (4.7) that $\mathbf{z}$ is independent of the cutoff $\chi$.

We now turn to the proof of (4.14). By (4.9),

$$
\begin{aligned}
0 & =\int L\left(F h\left(z_{\chi, \delta}^{\mu}(\omega)\right) d Q\right. \\
& =\int(L F) h\left(z_{\chi, \delta}^{\mu}(\omega)\right) d Q+\int F L h\left(z_{\chi, \delta}^{\mu}(\omega)\right) d Q .
\end{aligned}
$$

The first term converges to $\int L F h\left(z_{\chi}^{\mu}(\omega)\right) d Q$ as $\delta \rightarrow 0$. We only have to show that the second term converges to zero as $\delta \rightarrow 0$. Clearly, it suffices to show that as $\delta \rightarrow 0$,

$$
\int\left|L z_{\chi, \delta}^{\mu}\right| d Q \rightarrow 0, \quad \mu=0, \ldots, 4
$$

By definition,

$$
L z_{\chi, \delta}^{0}=\delta \delta^{3} \sum_{\alpha, i} \chi_{i}\left(\delta x_{\alpha}\right) p_{\alpha}^{i}
$$

Hence $\int\left|L z_{\chi, \delta}^{0}\right| d Q \rightarrow 0$ as $\delta \rightarrow 0$. Similarly,

$$
\begin{aligned}
L z_{\chi, \delta}^{i}= & \delta \delta^{3} \sum_{\alpha, j} \chi_{j}\left(\delta x_{\alpha}\right) p_{\alpha}^{2} \phi_{j}\left(p_{\alpha}\right) \\
& -\delta^{3} \sum_{\alpha \neq \beta} \chi\left(\delta x_{\alpha}\right) V_{i}\left(x_{\alpha}-x_{\beta}\right), \quad i=1,2,3 .
\end{aligned}
$$

The first term clearly goes to zero as $\delta \rightarrow 0$. As for the second term we use the antisymmetry of $V_{i}$ to write it as

$$
\frac{1}{2} \delta^{3} \sum_{\alpha \neq \beta} V_{i}\left(x_{\alpha}-x_{\beta}\right)\left[\chi\left(\delta x_{\alpha}\right)-\chi\left(\delta x_{\beta}\right)\right] .
$$

Since $\chi$ is smooth with compact support

$$
\left|\chi\left(\delta x_{\alpha}\right)-\chi\left(\delta x_{\beta}\right)\right| \leq \operatorname{const} \delta\left[1\left(\left|x_{\alpha}\right| \leq \delta^{-1} C\right)+1\left(\left|x_{\beta}\right| \leq \delta^{-1} C\right) \mid,\right.
$$

for some constant $C$. So (4.19) is bounded by

$$
\text { const } \delta \cdot \delta^{3} \sum_{\alpha \neq \beta} W\left(x_{\alpha}-x_{\beta}\right) 1\left(\left|x_{\alpha}\right| \leq \delta^{-1} C\right),
$$

where $W$ is a smooth positive function with compact support. By (4.6) we can bound the expectations of (4.18) by const $\delta$. Hence

$$
\lim _{\delta \rightarrow 0} \int\left|L z_{\chi, \delta}^{\imath}\right| d Q=0, \quad i=1,2,3 .
$$


Finally we have to bound $\int\left|L z_{\chi, \delta}^{0}\right| d Q$,

$$
\begin{aligned}
E^{Q}\left[\left|L z_{\chi, \delta}^{0}\right|\right]= & E^{Q}\left[\left|\delta \delta^{3} \sum_{i, \alpha} \chi_{i}\left(\delta x_{\alpha}\right) \phi_{i}\left(p_{\alpha}\right) \phi\left(p_{\alpha}\right)\right|\right] \\
& +E^{Q}\left[\left|\delta^{3} \sum_{\alpha \neq \beta} \sum_{\imath} \chi\left(\delta x_{\alpha}\right) \phi_{i}\left(p_{\alpha}\right) V_{i}\left(x_{\alpha}-x_{\beta}\right)\right|\right] .
\end{aligned}
$$

The first term on the right side of (4.19) is easy as before. The second term is trickier. Let $w_{i}(\mathbf{z})$ and $\sigma_{i}(\mathbf{t})$ denote the expectation and variance of $\phi_{i}\left(p_{\alpha}\right)$ with respect to $Q$ conditioned on $\mathbf{z}$. Note that since the extremal measures of momenta distribution are characterized by (4.8), $w_{i}$ and $\sigma_{i}$ depend on $\mathbf{z}$ only and are independent of the other parameter or structure of $Q$. We can now bound the second term of (4.19) by

$$
\begin{aligned}
E^{Q}\left[\left|\delta^{3} \sum_{i} \sum_{\alpha \neq \beta} \chi\left(\delta x_{\alpha}\right) \phi_{i}\left(p_{\alpha}\right) V_{i}\left(x_{\alpha}-x_{\beta}\right)\right|\right] \\
=E^{Q}\left[\left|\delta^{3} \sum_{i} \chi\left(\delta x_{\alpha}\right)\left[\phi_{i}\left(p_{\alpha}\right)-w_{i}\right] \sum_{\beta \neq \alpha} V_{i}\left(x_{\alpha}-x_{\beta}\right)\right|\right] \\
\quad+E^{Q}\left[\left|\delta^{3} \sum_{\alpha \neq \beta} \sum_{i} \chi\left(\delta x_{\alpha}\right) V_{i}\left(x_{\alpha}-x_{\beta}\right) w_{i}\right|\right] .
\end{aligned}
$$

The second term of (4.20) can be bounded as before. Using the Schwartz inequality the first term can be bounded by

$$
\begin{gathered}
E^{Q}\left\{\sum_{i=1}^{3} \sigma_{i}(\mathbf{z}) \delta^{3} E^{Q}\left[\sum_{\alpha} \chi\left(\delta x_{\alpha}\right)^{2}\left(\sum_{\beta} V_{i}\left(x_{\alpha}-x_{\beta}\right)\right)^{2} \mid \mathbf{z}\right]^{1 / 2}\right\} \\
\leq \operatorname{const} \delta^{3} E^{Q}\left[\sum_{i=1}^{3} \sum_{\alpha} \chi\left(\delta x_{\alpha}\right)^{2}\left(\sum_{\beta \neq \alpha} V_{i}\left(x_{\alpha}-x_{\beta}\right)\right)^{2}\right]^{1 / 2},
\end{gathered}
$$

where we use the boundedness of $\sigma_{\imath}(z)$. To bound the second expectation, let us divide the set $\left\{x|| x \mid \leq 2 \delta^{-1}\right\}$ into boxes of size $2 R$ with $R$ denoting the range of $V$. Let $\sigma$ index the boxes and let $N_{\sigma}$ be the number of particles in the $\sigma$ box.

$$
\delta^{3} E^{Q}\left[\sum_{i} \sum_{\alpha} \chi\left(\delta x_{\alpha}\right)^{2}\left(\sum_{\beta} V_{i}\left(x_{\alpha}-x_{\beta}\right)\right)^{2}\right]^{1 / 2} \leq \operatorname{const} \delta^{3} E^{Q}\left[\sum_{\sigma} N_{\sigma}^{3}\right]^{1 / 2}:
$$

By convexity and the inequality $\left(\sum_{\sigma} N_{\sigma}^{3}\right)^{1 / 3} \leq\left(\sum_{\sigma} N_{\sigma}^{2}\right)^{1 / 2}$ we see that (4.21) is
bounded by

$$
\text { const } \delta^{3} E^{Q}\left[\left(\sum_{\sigma} N_{\sigma}^{2}\right)^{3 / 4}\right] \leq \delta^{3}\left\{E^{Q}\left[\sum_{\sigma} N_{\sigma}^{2}\right]\right\}^{3 / 4} \text {. }
$$

By (4.7), $E^{Q}\left[\sum_{\sigma} N_{\sigma}^{2}\right]$ is bounded by $\delta^{-3}$ and (4.22) is bounded by const $\delta^{3 / 4}$. We have thus concluded that (4.17) vanishes as $\delta \rightarrow 0$ and thus Lemma 4.9.

By Lemma 4.6 we can represent the limit point $Q$ as

$$
Q=\int \beta(d \mathbf{z}) \nu(d \mathbf{x} \mid \mathbf{z}) \pi(d \mathbf{p} \mid \mathbf{z})
$$


where $\pi$ is defined in (4.3) with $\lambda$ chosen in such a way as to have average momenta and kinetic energy given by $\mathbf{z}$. Furthermore Lemmas 4.7 and 4.8 imply that

$$
\int L F(\omega) \nu(d \mathbf{x} \mid \mathbf{z}) \pi(d \mathbf{p} \mid \mathbf{z})=0
$$

for almost all $\mathbf{z}$. From (4.24) and the translation invariance of $Q, \nu \otimes \pi$ is a Gibbs state satisfying the DLR equation. We state this as the following.

Lemma 4.10. Any extremal limit point of the family $\left\{\bar{Q}_{\varepsilon}\right\}$ defined in (4.1) is a Gibbs state. It is characterized by the five parameters $z^{\mu}, \mu=0, \ldots, 4$.

Proof. By the translation invariance of $\nu(d \mathbf{x} \mid \mathbf{z})$ we can rewrite (4.24) as

$$
\int\left[\sum_{\alpha} \sum_{\imath}\left(\phi_{i}\left(p_{\alpha}\right)-w_{i}(\mathbf{z})\right) \frac{\partial F}{\partial x_{\alpha}^{i}}-\sum_{\beta \neq \alpha} \sum_{i} V_{i}\left(x_{\alpha}-x_{\beta}\right) \frac{\partial F}{\partial p_{\alpha}^{i}}\right] \nu(d \mathbf{x} \mid \mathbf{z}) \pi(d \mathbf{p} \mid \mathbf{z})=0,
$$

where $w_{i}(\mathbf{z})$ is the expectation of $\phi_{i}\left(p_{\alpha}\right)$ with respect to $\pi(d \mathbf{p} \mid \mathbf{z})$ (as in the proof of Lemma 4.9). Then the proof follows easily along the line of the proof of Lemma 4.5 in [OV]. In fact the particular form of $\phi$ does not play any role here.

Now the local ergodic Theorem 3.10 is just a simple corollary of Lemma 4.10. By taking the limit $\varepsilon \rightarrow 0$ we only have to check that

$$
\varlimsup_{k \rightarrow \infty} \sup _{Q \in A} E^{Q}\left[\left|F_{k}(\omega)-\hat{F}\left(\zeta_{k}(\omega)\right)\right| \sigma_{k}(\omega)\right]=0,
$$

where $A$ denotes the set of limit points of $\bar{P}_{\varepsilon}$. The characteristic function $\sigma_{k}$ reduces the problem to verification of (4.25) with conserved quantities in the single phase region of Gibbs states with potential $V$ and kinetic energy $\phi(p)$. Combining with Lemma 4.10 we only have to check (4.25) for Gibbs states in the single phase region. This is simply the law of large numbers for Gibbs states.

\section{Large Deviations for Gibbs Point Processes}

We will develop here a large deviation theory for Gibbs point processes, needed for the proof of Theorem 2.1 and Corollary 2.2. For Gibbs processes on a lattice this theory has already been developed in $[\mathrm{O}]$ and $[\mathrm{Re}]$. For an account of general theory of large deviation, see [V1].

Initially we want to develop a large deviation theory for a Poisson point process $P$ on $\mathbb{R}^{3} \times \mathbb{R}^{3}$. The intensity of the Poisson process will be the measure $d x \times f(p) d p$. The density $f(p)$ will be nonnegative and $\int \mathbb{R}^{3} f(p) d p=1$. A point of $\mathbb{R}^{3} \times \mathbb{R}^{3}$ will be denoted by $(x, p)$ and the sample space $\Omega$ will consist of points $\omega$, denoting a configuration $\left\{\left(x_{\alpha}, p_{\alpha}\right)\right\}$. Any bounded region $B$ in $\mathbb{R}^{3}$ will have only a finite number of $x_{a}$ in it and one can think of $p_{\alpha}$ as tags and consider the corresponding finite configuration in $B \times \mathbb{R}^{3}$. The configuration $x_{\alpha}$ alone will constitute a Poisson point process on $\mathbb{R}^{3}$ with intensity given by the Lebesgue measure. The Poisson measure $P$ on $\Omega$ is invariant with respect to the group of $x$-translation $\tau_{x}$. Suppose $Q$ is any other $\tau_{x}$-invariant point process measure on $\Omega$. Let $D_{l}$ be a cube of side $l$ in $\mathbb{R}^{3}$; we can define the relative entropy $H_{l}(Q \mid P)$ of the restriction of $Q$ to $D_{l} \times \mathbb{R}^{3}$, with respect to 
the same restriction of $P$. By restriction of course we mean restriction to the $\sigma$-field $\mathscr{F}_{l}$ generated by configurations in that domain. We define then

$$
H_{l}(Q \mid P)=\sup _{F_{l}}\left\{E^{Q}\left(F_{l}\right)-\log E^{P}\left(e^{F_{l}}\right)\right\}
$$

where the sup is taken over all the bounded continuous $\mathscr{F}_{l}$-measurable functions $F_{l}$ on $\Omega$. Because of independence properties and stationarity of $P, H_{l}$ is superadditive and one can define the entropy density as

$$
h(Q / P)=\lim _{\delta \rightarrow \infty} \frac{1}{l^{3}} H_{l}(Q \mid P)=\sup _{l} \frac{1}{l^{3}} H_{l}(Q \mid P) .
$$

To any configuratioin $\omega \in \Omega$, we can associate a $\sigma$-finite measure on $\mathbb{R}^{3} \times \mathbb{R}^{3}$ by counting the number of points $\left(x_{\alpha}, p_{\alpha}\right)$ in any given set. Then the space $\Omega$ can be made into a topological space under vague convergence. That is to say that for any test function $\phi(x, p)$ which is continuous and has compact support in $\mathbb{R}^{3} \times \mathbb{R}^{3}$, the function on $\Omega$

$$
\sum_{\alpha} \phi\left(x_{\alpha}, p_{\alpha}\right)
$$

is continuous on $\Omega$. This in turn provides a weak convergence definition for the space $\mathscr{l} b$ of stochastic point processes on $\Omega$ and we shall limit ourselves mainly to the $\tau_{x}$-invariant ones $\mathscr{C l}_{0}$.

Let $N_{B}(\omega)$ be the number of points of the configuration $\omega$ in $B \times \mathbb{R}^{3}$, where $B$ is a bounded set in $\mathbb{R}^{3}$. For any stationary point process $Q$, if for any bounded $B \subset \mathbb{R}^{3}$

$$
E^{Q}\left[N_{B}\right]<+\infty
$$

then there exists a positive $\sigma$-finite measure $q(d x, d p)$ on $\mathbb{R} \times \mathbb{R}^{3}$ such that

$$
E^{Q}\left[N_{B}\right]=\int_{B \times \mathbb{R}^{3}} q(d x, d p) .
$$

By stationarity we have

$$
q(d x, d p)=\varrho d x \cdot \pi(d p),
$$

where $\varrho$ is a constant density of the $x$-configurations and $\pi$ is the distribution of the " $p_{\alpha}$."

Theorem 5.1. (i) $h(Q \mid P)$ is lower semicontinuous.

(ii) for any finite number $l$, the set $\{Q: h(Q \mid P) \leq l\}$ is compact.

(iii) if $Q_{n} \rightarrow Q$ with $h\left(Q_{n} \mid P\right) \leq c$ for any $n$, then $q_{n}(d x, d p)=\varrho_{n} d x \cdot \pi_{n}(d p)$ converges to $q(d x, d p)=\varrho d x \cdot \pi(d p)$ of the limiting $Q$.

Proof. (i): The lower semicontinuity of $H_{l}$ follows easily from this definition and, by (5.1), $h(Q)$ is also lower semicontinuous.

(ii) and (iii): By the entropy inequality it is easy to show that if $Q \in \mathrm{M}_{0}$ and $h(Q \mid P) \leq c$, then for any bounded $B \subset \mathbb{R}^{3}$ there exists a constant $c_{B}$,

$$
E^{Q}\left(N_{B} \log N_{B}\right) \leq c_{B} .
$$

Then (ii) and (iii) follow by standard arguments.

The following Theorems 5.2 and 5.3 are the large deviation theory relative to a Poisson point process. For the upper bound contained in Theorem 5.2 let us assume 
that $F(\omega)$ is a local function measurable with respect to configurations on some $B \times \mathbb{R}^{3}$ and satisfies a bound

$$
F(\omega) \leq c_{1}+c_{2} N_{B_{0}}(\omega)
$$

for some constant $c_{1}, c_{2}$.

Theorem 5.2. Under the above assumption

$$
\limsup _{l \rightarrow \infty} \frac{1}{l^{3}} \log E^{P}\left[\exp \int_{D_{l}} F\left(\tau_{x} \omega\right) d x\right] \leq \sup _{Q \in \mathscr{H}_{0}}\left[E^{Q}[F]-h(Q \mid P)\right]
$$

and the right-hand side is finite.

Proof. Let us first assume that $F(\omega)$ is bounded in absolute value. Then by the entropy inequality we have

$$
\log E^{P}\left[\exp \int_{D_{l}} F\left(\tau_{x} \omega\right) d x\right]=\sup _{Q^{\prime}}\left\{E^{Q^{\prime}}\left[\int_{D_{l}} F\left(\tau_{x} \omega\right) d x\right]-H_{l+l_{0}}\left(Q^{\prime} \mid P\right)\right\},
$$

where the supremum is taken over all point processes $Q^{\prime}$ on $D_{l+l_{0}} \times \mathbb{R}^{3}$. Here we are assuming that $B_{0} \subset D_{l_{0}}$. We extend the measure $Q^{\prime}$ where the supremum is attained to a point process on $\mathbb{R}^{3} \times \mathbb{R}^{3}$ by taking independent copies on all disjoint translated cubes of $\mathbb{R}^{3} \times \mathbb{R}^{3}$. The measure $Q^{\prime \prime}$ so obtained is not stationary but can be made stationary by defining

$$
\hat{Q}_{l+l_{0}}=\frac{1}{\left(l+l_{0}\right)^{3}} \int_{D_{l+l_{0}}} \tau_{x} Q^{\prime \prime} d x .
$$

By (5.2), convexity of $H_{l}$ and independence properties of the Poisson measure $P$ we have

$$
\begin{aligned}
h\left(\hat{Q}_{l+l_{0}} \mid P\right) & =\lim _{n \rightarrow \infty} \frac{1}{\left(n\left(l+l_{0}\right)\right)^{3}} H_{n\left(l+l_{0}\right)}\left(\hat{Q}_{l+l_{0}} \mid P\right) \\
& \leq \lim _{n \rightarrow \infty} \frac{1}{\left(n\left(l+l_{0}\right)\right)^{3}} \frac{1}{\left(l+l_{0}\right)^{3}} \int_{D_{l+l_{0}}} H_{n\left(l+l_{0}\right)}\left(\tau_{x} Q^{\prime \prime} \mid P\right) d x \\
& \leq \frac{1}{\left(l+l_{0}\right)^{3}} H_{l+l_{0}}\left(Q^{\prime \prime} \mid P\right) \\
& =\frac{1}{\left(l+l_{0}\right)^{3}} H_{l+l_{0}}\left(Q^{\prime} \mid P\right) .
\end{aligned}
$$

Furthermore we have

$$
\left|E^{\hat{Q}_{l}}(F(\omega))-\frac{1}{l^{d}} E^{Q^{\prime}}\left(\int_{D_{l}} F\left(\tau_{x} \omega\right) d x\right)\right| \leq\|F\|_{\infty} \cdot o(1)
$$

as $l \rightarrow \infty$. This proves the upper bound for bounded $F$.

Now assume $F$ only bounded above and define $F_{k}=\max \{F,-k\}$. Then we have

$$
\limsup _{l \rightarrow \infty} \frac{1}{l^{3}} \log E^{P}\left(\exp \int_{D_{e}} F\left(\tau_{x} \omega\right) d x\right) \leq \sup _{Q \in \mathscr{H}_{0}}\left\{E^{Q}\left[F_{k}(\omega)\right]-h(Q \mid P)\right\}=\sigma_{k} .
$$


We need only to establish $\lim _{k \rightarrow \infty} \sigma_{k} \leq \sup _{Q \in M_{0}}\left\{E^{Q}(F)-h(Q \mid P)\right\}$ (the limit exists since $\sigma_{k}$ is decreasing.) If $\lim _{k \rightarrow \infty} \sigma_{k}=-\infty$ there is nothing to prove. Let us assume $\lim _{k \rightarrow \infty} \sigma_{k}=\sigma>-\infty$. Since $F_{k}$ is bounded above we can find $Q_{k}$ such that $h\left(Q_{k} \mid P\right)$ is bounded and $E^{Q_{k}}\left[F_{k}\right]-h\left(Q_{k} \mid P\right) \rightarrow \sigma$. The boundedness of $h\left(Q_{k} \mid P\right)$ means that along some subsequence we will have a weak limit $Q$ and moreover $Q_{k} \rightarrow Q$ strongly, i.e. for each Borel set in the $\sigma$-field corresponding to every $B \times \mathbb{R}^{d}$. In particular

$$
E^{Q_{k}}(F) \rightarrow E^{Q}(F)
$$

for every bounded measurable $F$. Therefore

$$
\lim _{k \rightarrow \infty} E^{Q_{k}}\left(F_{k}\right) \leq E^{Q}(F)
$$

The lower semicontinuity of $h(Q \mid P)$ completes the argument.

For removing the upper bound on $F$ we replace $F$ by $F_{k}=\min (F, k)$ and we will use the fact that $E^{P} e^{\lambda N_{B_{0}}}<+\infty$ for every $\lambda<+\infty$. By Hölder's inequality if $\frac{1}{p}+\frac{1}{q}=1$ we can bound

$$
\begin{aligned}
E^{P}\left\{\exp \left[\int_{D_{e}} F\left(\tau_{x} \omega\right) d x\right]\right\} \leq & \left(E^{P}\left\{\exp \left[p \int_{D_{e}} F_{k}\left(\tau_{x} \omega\right) d k\right]\right\}\right)^{1 / p} \\
& \times\left(E^{P}\left\{\exp \left[q \int_{D_{e}}\left(F-F_{k}\right)\left(\tau_{x} \omega\right) d x\right]\right\}\right)^{1 / q} \\
= & T_{1} \cdot T_{2} .
\end{aligned}
$$

If we take $\log$ and divide by $1 / l^{3}$ we get for the first term

$$
\limsup _{l \rightarrow \infty} \frac{1}{l^{3}} \log T_{1} \leq \frac{1}{p} \sup _{Q \in \mathscr{H}_{0}}\left\{E^{Q}[p F]-h(Q \mid P)\right\}
$$

while the second term is estimated by

$$
T_{2} \leq E^{P}\left\{\exp \left[q \int_{D_{e}}\left[c_{2} N_{B_{0}}\left(\tau_{x} \omega\right)+c_{1}-k\right]^{+} d x\right]\right\}^{1 / q}
$$

We can think of $D_{l}$ of disjoint union of translates of $B_{0}$ and if we denote by

$$
\xi_{x}(\omega)=\sum_{\alpha}^{l^{3} / l_{0}^{3}}\left[c_{2} N_{D_{l_{0}}}\left(\tau_{x+\alpha} \omega\right)-\left(k-c_{1}\right)\right]^{+},
$$


then we can bound $T_{2}$ by (using Jensen's inequality)

$$
\begin{aligned}
T_{2} & \leq E^{P}\left\{\exp \left[q \int_{D_{l_{0}}} \xi_{x} d x\right]\right\}^{1 / q} \\
& \leq\left(\frac{1}{l_{0}^{3}} \int_{D_{l_{0}}} E^{P}\left\{\exp \left[q l_{0}^{3} \xi_{x}\right]\right\}\right)^{1 / q} \\
& =\left(E^{P}\left\{\exp \left(q l_{0}^{3}\left[c_{2} N_{D_{l_{0}}}(\omega)-k-c_{1}\right)\right]^{+}\right)\right)^{\left(l^{3} / l_{0}^{3}\right) \cdot(1 / q)} .
\end{aligned}
$$

We obtain then

$$
\limsup _{l \rightarrow \infty} \frac{1}{l^{3}} \log T_{2} \leq \frac{1}{l_{0}^{3}} \frac{1}{q} \log E^{P}\left[\exp \left(q l_{0}^{3}\left[c_{2} N_{D_{l_{0}}}(\omega)-\left(k-c_{1}\right)\right]^{+}\right)\right] .
$$

Let $k \rightarrow \infty$, then the right-hand side goes to zero. Now we let $p \rightarrow 1$ and we get the right bound.

For the following lower bound we can assume $F(\omega)$ to be an arbitrary function measurable with respect to the configurations on some $D_{l_{0}} \times \mathbb{R}^{3}$. Then we can prove

\section{Theorem 5.3.}

$$
\operatorname{liminv}_{l \rightarrow \infty} \frac{1}{l^{3}} \log E^{P}\left(\exp \left[\int_{D_{l}} F\left(\tau_{x} \omega\right) d x\right]\right) \geq \sup _{Q \in \mathscr{M}_{0}}\left[E^{Q}(F)-h(Q \mid P)\right],
$$

where the sup is taken over all $Q$ such that $h(Q \mid P)<+\infty$ and $E^{Q}\left(F^{-}\right)<+\infty$.

Proof. We can take any permissible $Q$ and since $h(Q \mid P)<\infty$ we know that

$$
\left.\frac{d Q}{d P}\right|_{F_{D_{l+l_{0}} \times \mathbb{R}^{3}}}=\psi_{l+l_{0}}
$$

exists. Then by Jensen's inequality

$$
\begin{aligned}
E^{P}\left[\exp \left(\int_{D_{l}} F\left(\tau_{x} \omega\right) d x\right)\right] & =E^{Q}\left[\exp \left(\int_{D_{l}} F\left(\tau_{x} \omega\right) d x-\log \psi_{l+l_{0}}\right)\right] \\
& \geq \exp \left[E^{Q}\left(\int_{D_{l}} F\left(\tau_{x} \omega\right) d x\right)-H_{l+l_{0}}(Q \mid P)\right] \\
& \geq \exp \left[l^{3} E^{Q}(F)-\left(l+l_{0}\right)^{3} h(Q \mid P)\right] .
\end{aligned}
$$

Taking log, dividing by $l^{3}$ and letting $l \rightarrow \infty$ we obtain the lower bound.

Remark. The above proof can be easily modified to yield a lower bound for

$$
\operatorname{liminv}_{l \rightarrow \infty} \frac{1}{l^{3}} \log E^{P}\left(\chi_{l} \exp \left[\int_{D_{l}} F\left(\tau_{x} \omega\right) d x\right]\right) \geq \sup _{Q \in A_{e}}\left[E^{Q}(F)-h(Q \mid P)\right],
$$


where $\chi_{l}$ is defined by

$$
\chi_{l}=\left\{\omega: \frac{1}{D_{l}} \int_{D_{l}} G\left(\tau_{x} \omega\right) d x \in(a, b)\right\} \text { for some }-\infty<a<b<\infty
$$

and

$$
A_{e}=\left\{Q: \int G(\omega) d Q \in(a, b), Q \text { ergodic }\right\}
$$

and by arguments standard in large deviation theory we can replace $A_{e}$ by

$$
A=\left\{Q: \int G(\omega) d Q \in(a, b)\right\} .
$$

We now turn our attention to a similar problem for a Poisson point process $P_{\gamma}$ with intensity $\gamma d x \times f(p) d p$ on $T^{3} \times \mathbb{R}^{3}$ as $\gamma \rightarrow \infty$. We will have a family $F(x, \omega)$ of functions depending on a fixed domain $D_{l_{0}} \times \mathbb{R}^{3}$ in $\mathbb{R}^{3} \times \mathbb{R}^{3}$ and depending in some continuous manner on $x \in T^{3}$ as a parameter. We will think of $\gamma=\varepsilon^{-3}$, and $\varepsilon \rightarrow 0$ as $\gamma \rightarrow \infty$. If $x$ is any point in $T^{3}$ we can define $\omega_{x, \varepsilon}$, a configuration in $\mathbb{R}^{3} \times \mathbb{R}^{3}$ in the same fashion as in Sect. 3. Then if $\varepsilon$ is small enough $F\left(x, \omega_{x, \varepsilon}\right)$ is well defined. We will be interested in the behavior of

$$
\frac{1}{\gamma} \log E^{P_{\gamma}}\left[\exp \left\{\gamma \int_{T^{3}} F\left(x, \omega_{x, \varepsilon}\right) d x\right\}\right]
$$

as $\gamma \rightarrow \infty$. Remember that $\gamma=\varepsilon^{-3}$.

Theorem 5.4. Assume that $F(\omega)$ is a bounded function measurable on $D_{l_{0}} \times \mathbb{R}^{3}$. Then

$$
\lim _{\gamma \rightarrow \infty} \frac{1}{\gamma} \log E^{P_{\gamma}}\left[\exp \left\{\gamma \int_{T^{3}} F\left(\omega_{x, \varepsilon}\right) d x\right\}\right]=\sup _{Q \in \mathscr{H}_{0}}\left[E^{Q}[F]-h(Q \mid P)\right] .
$$

Proof. It is no different from that of Theorems 5.2 and 5.3. If we ignore strips of size $\varepsilon$, adding up to a volume $O\left(\varepsilon^{-2}\right)$ and expand the rest out to a cube of side nearly $1 / \varepsilon$ we are back to the earlier case.

We now consider integrals of the form

$$
Z(\gamma)=E^{P_{\gamma}}\left\{\exp F_{\gamma}(\omega)\right\}
$$

where $\left(\varepsilon^{-3}=\gamma\right)$

$$
\begin{aligned}
F_{\gamma}(\omega)= & \sum_{\alpha} A_{0}\left(x_{\alpha}\right)+\sum_{\alpha} \sum_{i=1}^{3} A_{i}\left(x_{\alpha}\right) p_{\alpha}^{2}-\frac{1}{2} \sum_{\alpha} A_{4}\left(x_{\alpha}\right) \sum_{\beta \neq \alpha} V\left(\frac{x_{\alpha}-x_{\beta}}{\varepsilon}\right) \\
& -\sum_{\alpha} A_{5}\left(x_{\alpha}\right) \phi\left(p_{\alpha}\right)+\gamma \int_{T^{3}} G\left(x, \omega_{x, \varepsilon}\right) d x .
\end{aligned}
$$

We make the following assumptions:

1) $A_{\jmath}, j=0, \ldots, 5$ are continuous functions of $x \in T^{3}$.

2) $V(x)$ is a continuous function with compact support in $\mathbb{R}^{3}$ such that $V=V_{1}+V_{2}$ with $V_{1}(x) \geq 0, V_{1}(0)>0$, and $V_{2}$ being a positive definite potential. 
3) $\sum_{i=1}^{3} A_{i}(x) p^{i} \leq A_{5}(x) \phi(p)$ for all $x$ and $p$.

4) $G(x, \omega)=\sum_{s} g_{s}(x) G_{s}(\omega)$, where $g_{s}(x)$ are bounded measurable functions depending on configurations in some fixed domain $D_{l_{0}} \times \mathbb{R}^{3}$.

5) $A_{4}(x) \geq a_{0}>0$.

Theorem 5.5. Under the above assumptions

$$
\lim _{\gamma \rightarrow \infty} \frac{1}{\gamma} \log Z(\gamma)=\int_{T^{3}} \sup _{Q \in \mathscr{H}_{0}}\left[E^{Q}\left(\hat{F}_{x}(\omega)\right)-h(Q \mid P)\right] d x,
$$

where

$$
\hat{F}_{x}(\omega)=\sum_{i=0}^{5} A_{i}(x) \Psi_{i}(\omega)+\sum_{s} g_{s}(x) G_{s}(\omega)
$$

and

$$
\begin{aligned}
\Psi_{0}(\omega) & =\sum_{\alpha} u\left(x_{\alpha}\right), \\
\Psi_{i}(\omega) & =\sum_{\alpha} u\left(x_{\alpha}\right) p_{\alpha}^{i}, \quad i=1,2,3, \\
\Psi_{4}(\omega) & =-\frac{1}{2} \sum_{\alpha} u\left(x_{\alpha}\right) V\left(x_{\alpha}-x_{\beta}\right), \\
\Psi_{5}(\omega) & =-\sum_{\alpha} u\left(x_{\alpha}\right) \phi\left(p_{\alpha}\right) .
\end{aligned}
$$

Here $u$ is a positive function of compact support supported in $D_{l_{0}}$ with $\int_{\mathbb{R}^{3}} u(x) d x=1$.

Remark. The assumption that $V$ is bounded and is of finite range is not essential. The proof we give in the following is taken from [CLY] where one needs to control Coulomb potential. We prefer to keep our proof in a simple form.

Proof. The proof is just an easy step away from Theorems 2, 3, and 4. First let us remark that we can find approximations for $A_{2}(x)$ and $g_{i}(x)$ that are constant on some cubic partition of $T^{3}$, both from above and below, and we can assume without loss of generality that all these functions are piecewise constant.

The only restriction on the function $u$ is that it has compact support, is nonnegative and is of total integral one. It is not hard to see that by the stationarity of $Q$ the variational formula gives the same value independent of the choice of $u$ (subjected to the previous conditions, of course). In the following we prove Theorem 5.5 with $u$ being a characteristic function of a cube.

Divide $T^{3}$ into disjoint boxes $B_{\sigma}$ of size $\varepsilon l$. We can translate the boxes by $\tau$ with $0 \leq \tau_{i} \leq \varepsilon l, i=1,2,3$. Let $F_{\sigma+\tau}(\omega)$ denote the contributions of $F_{\gamma}$ in the box $B_{\sigma}$ translated by $\tau$. There are ambiguities in this definition which we shall clarify as follows. First of all we can assume $A_{\jmath}$ is constant in the box $\alpha+\tau$ and replace $A_{\jmath}\left(x_{\alpha}\right)$ by $A_{j}(\sigma+\tau)$. Next $V\left(x_{\alpha}-x_{\beta} / \varepsilon\right)$ is replaced by

$$
V\left(x_{\alpha}-x_{\beta} / \varepsilon\right) 1_{\sigma+\tau}\left(x_{\alpha}\right) 1_{\sigma+\tau}\left(x_{\beta}\right),
$$


namely, we neglect the interactions between neighboring boxes. Certainly we made certain errors. It's not hard to see that there is a bounded function with compact support $W$ such that

$$
F(\omega) \leq(\varepsilon l)^{-3} \int d \tau \sum_{\sigma} F_{\sigma+\tau}(\omega)+l^{-1} \sum_{\alpha \neq \beta} W\left(x_{\alpha}-x_{\beta} / \varepsilon\right) .
$$

The last term is in general of order $N^{2}$ but one can control it in the following way. Let

$$
F^{(\theta)}=F+\theta \sum_{\beta \neq \alpha} V\left(x_{\alpha}-x_{\beta} / \varepsilon\right) .
$$

Apply previous bound to $F^{(\theta)}$,

$$
\begin{aligned}
F(\omega) & \leq(\varepsilon l)^{-3} \int d \tau \sum_{\sigma}\left(F^{(\theta)}\right)_{\sigma+\tau}(\omega)+\Omega, \\
\Omega & =\sum_{\alpha \neq \beta} l^{-1}(1+\theta) W\left(x_{\alpha}-x_{\beta} / \varepsilon\right)-\theta V\left(x_{\alpha}-x_{\beta} / \varepsilon\right) .
\end{aligned}
$$

By assumption $V=V_{1}+V_{2}$ with $V_{2}$ positive definite and $V_{1}$ nonnegative with $V_{1}(0)>0$. By Lemma 5.7 below, for $\ell$ large enough there exists a $\delta>0$ such that:

$$
\Omega \leq \delta^{-1} l^{-1} N-\theta \sum_{\alpha \neq \beta} V_{2}\left(x_{\alpha}-x_{\beta} / \varepsilon\right) \leq\left(\delta^{-1} l^{-1}+\text { const } \theta\right) N .
$$

Hence

$$
\begin{aligned}
\log Z(\gamma) \leq & \left(\delta^{-1} l^{-1}+\text { const } \theta\right) N \\
& +\log E^{P_{\gamma}}\left[\exp A_{v_{r}} \sum\left(F^{(\theta)}\right)_{\sigma+\tau}(\theta)\right] .
\end{aligned}
$$

By Jensen's inequality the last expectation is bounded by

$$
A v_{\tau} \log E^{P_{\gamma}}\left[\exp \left(\sum\left(F^{(\theta)}\right)_{\sigma+\tau}(\omega)\right)\right] .
$$

Note that for $\tau$ fixed there is no interaction between neighboring boxes. So the last expectation is nothing but

$$
A v_{r} \sum_{\sigma+\tau} E^{P_{\gamma}}\left[\exp \left(\left(F^{(\theta)}\right)_{\sigma+\tau}(\omega)\right)\right] .
$$

We can now apply Theorem 5.2 and let $l \rightarrow \infty$ and then $\theta \rightarrow 0$ to conclude our upper bound.

The lower bound is much easier. First let us divide $T^{3}$ into disjoint cubes of size $\varepsilon l$. Let $A_{\alpha}$ denote such a typical cube and let $B_{\alpha} \subset A_{\alpha}$ be defined by

$$
B_{\alpha}=\left\{x \mid \operatorname{dist}\left(x, A_{\alpha}^{c}\right) \geq l_{0}\right\} .
$$

Since we need only lower bound, we can restrict to the set that no particle exists in the corridors $T^{3} \backslash \bigcup_{\alpha} B_{\alpha}$. The probability of such an event under $P_{\gamma}$ is $\exp [-C(l) N]$, where $C(l) \rightarrow 0$ as $l \rightarrow \infty$. Moreover, the integrand factors into product over cubes as no interaction presents in this event. We now use Theorem 5.3 for each cube and sum them up to the right side of Theorem 5.3. Note that the errors caused by 
restricting to the specific event mentioned above is negligible in the limit $\gamma \rightarrow \infty$ and $l \rightarrow \infty$.

Consider now the canonical partition function with the number of particles fixed at $N$ that goes to $\infty$ as $\varepsilon^{-3}$ :

$$
Z_{N}^{c}=E^{P_{N}}\left\{\exp \left[F_{\gamma}(\omega)\right]\right\}
$$

where $P_{N}$ is the measure on $\left(T^{3} \times \mathbb{R}^{3}\right)^{N}$ defined by

$$
d P_{N}=\prod_{\alpha=1}^{N} d x_{\alpha} \times f\left(p_{\alpha}\right) d p_{\alpha} .
$$

Then the previously defined [see (5.3)] grand canonical partition function is related to the canonical one by

$$
Z_{\gamma}=e^{-\gamma} \sum_{N=0}^{\infty} \frac{\gamma^{N}}{N !} Z_{N}^{c}
$$

Theorem 5.6. Suppose that $\lim _{N \rightarrow \infty} N \gamma^{-1}=1$. Then

$$
\lim _{N \rightarrow \infty} \frac{1}{N} \log Z_{N}^{c}=\sup _{\int_{T^{3}} \varrho\left(Q_{x}\right) d x=1}\left\{\int d x\left[E^{Q_{x}}\left\{F_{x}(\omega)\right\}-h\left(Q_{x} \mid P\right)\right]\right\}
$$

(here $\varrho(Q)$ is the density of $Q$ ).

Proof. If we consider

$$
Z_{\gamma}^{a, b}=\sum_{\gamma a<N<\gamma b} \frac{e^{-\gamma^{N}}}{N !} Z_{N}^{c},
$$

then the remark after Theorem 5.3 yields

$$
\begin{gathered}
\operatorname{liminv}_{\gamma \rightarrow \infty} \frac{1}{\gamma} \log Z_{\gamma}^{a, b} \geq \sup \left\{\int_{T^{3}} d x\left[E^{Q x}\left\{F_{x}(\omega)\right\}-h\left(Q_{x} \mid P\right)\right] ;\right. \\
\left.Q_{x}: a<\int_{T^{3}} \varrho\left(Q_{x}\right) d x<b\right\} .
\end{gathered}
$$

We note that

$$
Z_{N-K}^{c} \leq(\text { const })^{K} Z_{N}^{c}
$$

To see this, let us integrate out one particle, say $x_{1}$, in $Z_{N}^{c}$. For any distribution of $x_{2}, \ldots, x_{N}$ we can always find a set $A$ of measure at least $\alpha$ with $\alpha>0$ such that

$$
\sup _{x \in A} \sum_{j=2}^{N}\left|V\left(x-x_{j}\right)\right| \leq \text { const . }
$$

for some constant independent of $N$. By restricting $x_{1}$ integration to $A$ we get the desired bound for $k=1$. The general case follows by induction. 
Now let us choose $a=1-\delta$ and $b=1$ to have a lower bound for $Z_{\gamma}^{a, b}$. We obtain a lower bound for $Z_{N}^{c}$ by using the upper bound on $Z_{N-k}^{c}$. Finally let $\delta \rightarrow 0$ at the end to conclude the lower bound of Theorem 5.6.

To obtain the upper bound we define

$$
Z_{\gamma}^{\lambda}=\sum_{N=0}^{\infty} e^{-\gamma} \frac{\gamma^{N}}{N !} e^{\lambda N} Z_{N}^{c}
$$

Our methods can evaluate

$$
\limsup _{\gamma \rightarrow \infty} \frac{1}{\gamma} \log Z_{\gamma}^{\lambda}
$$

for any real $\lambda$. We then note that

$$
\lim _{N \rightarrow \infty} \frac{1}{N} \log \frac{e^{-N} N^{N}}{N !}=0 .
$$

Therefore

$$
\begin{aligned}
\limsup _{N \rightarrow \infty} \frac{1}{N} \log Z_{N}^{c} & \leq \limsup _{N \rightarrow \infty}\left[\frac{1}{N} \log Z_{N}^{\lambda}-\lambda\right] \\
& \leq \inf _{\lambda} \limsup _{N \rightarrow \infty}\left[\frac{1}{N} \log Z_{N}^{\lambda}-\lambda\right],
\end{aligned}
$$

which yields the right-hand side of our theorem.

Lemma 5.7. Suppose $W$ is a positive bounded function with compact support on $\mathbb{R}^{3}$. Then there is a $\delta>0$ such that

$$
\delta \sum_{\alpha \neq \beta}^{N} W\left(x_{\alpha}-x_{\beta}\right) \leq \sum_{\alpha \neq \beta}^{N} V\left(x_{\alpha}-x_{\beta}\right)+N .
$$

Proof. Proof of Lemma 4.2 in [V] generalized to more dimensions.

We will review now certain facts from thermodynamics of continuous systems, based partly on the large deviation theory developed earlier.

Suppose we are given a kinetic energy function $\phi(p)$ for $p \in \mathbb{R}^{3}$, which is continuous nonnegative, and growing at least linearly at $\infty$. We consider the set $L$ of $\left(\lambda_{1}, \lambda_{2}, \lambda_{3}, \lambda_{4}\right)$ in $\mathbb{R}^{3} \times \mathbb{R}_{+}$satisfying

$$
\int \exp \left[\sum_{i=1}^{3} \lambda_{i} p_{i}-\lambda_{4} \phi(p)\right] d p<+\infty .
$$

Let $L^{0}$ be the interior of $L$. Suppose $V$ is a pair potential of the class described earlier, then for any configuration $\left(x_{\alpha}, p_{\alpha}\right)$ in a bounded cube $D$ the grand canonical partition function is defined, for $\lambda=\left(\lambda_{0}, \lambda_{1}, \ldots, \lambda_{4}\right), \lambda_{0} \in \mathbb{R}$ and $\left(\lambda_{1}, \ldots, \lambda_{4}\right) \in L_{0}$, by:

$$
Z_{D}(\lambda)=\sum_{N=0}^{\infty} e^{-|D|} \frac{e^{\lambda_{0} N}}{N !} \int_{\left(D \times \mathbb{R}^{3}\right)^{N}} \ldots \int_{\alpha} \exp \left[\sum_{\alpha} \sum_{i=1}^{3} \lambda_{i} p_{\alpha}^{2}-\lambda_{4} \mathscr{H}_{N}\right] \prod_{\alpha=1}^{N} d x_{\alpha} d p_{\alpha}
$$

where

$$
\mathscr{H}_{N}=\frac{1}{2} \sum_{\alpha \neq \beta} V\left(x_{\alpha}-x_{\beta}\right)+\sum_{\alpha} \phi\left(p_{\alpha}\right)
$$


The pressure is defined by

$$
\psi(\lambda)=\lim _{D \nearrow \mathbb{R}^{3}} \frac{1}{|D|} \log Z_{D}(\lambda) .
$$

Although the existence of this limit can be proved in many ways we will use large deviation theory to see it. We can pick $\delta$ such that $\left(\lambda_{1}, \lambda_{2}, \lambda_{3}, \lambda_{4}-\delta\right) \in L^{0}$ and normalize $e^{-\delta \phi(p)}$ to be a probability density. We then see by large deviation theory that $\psi(\lambda)$ exists (Theorems 5.2 and 5.3, observe that the integrand is bounded above) and

$$
\psi(\lambda)=\sup _{Q \in \mathbb{H}_{0}}\left\{E^{Q}\left(F_{\lambda, \delta}\right)-h\left(Q \mid P_{\delta}\right)\right\}
$$

where (recall $u$ from Theorem 5.5)

$$
\begin{aligned}
F_{\lambda, \delta}= & \lambda_{0} \sum_{\alpha} u\left(x_{\alpha}\right)+\sum_{i=1}^{3} \sum_{\alpha} \lambda_{i} u\left(x_{\alpha}\right) p_{\alpha}^{i}-\frac{\lambda_{4}}{2} \sum_{(\alpha \neq \beta)} u\left(x_{\alpha}\right) V\left(x_{\alpha}-x_{\beta}\right) \\
& -\left(\lambda_{4}-\delta\right) \sum_{\alpha} u\left(x_{\alpha}\right) \phi\left(p_{\alpha}\right)+\left(\log c_{\delta}\right) \sum_{\alpha} u\left(x_{\alpha}\right) .
\end{aligned}
$$

Here $c_{\delta}=\int_{\mathbb{R}^{3}} e^{-\delta \phi(p)} d p$ and $u \geq 0$ is a compactly supported function with $\int_{\mathbb{R}^{3}} u(x) d x=$ 1. $P_{\delta}$ is the Poisson process with intensity $d x \times \exp (-\delta \phi(p)) d p /$ Normalization. It is easy to check that

$$
h\left(Q \mid P_{\delta}\right)-\delta E^{Q}\left[\sum_{\alpha} u\left(x_{\alpha}\right) \phi\left(p_{\alpha}\right)\right]-\left(\log c_{\delta}\right) E^{Q}\left(\sum_{\alpha} u\left(x_{\alpha}\right)\right)
$$

is independent of $\delta$ and defines a functional $s(Q)$. Then

$$
\psi(\lambda)=\sup _{Q \in \mathscr{H}_{0}}\left[E^{Q}\left[F_{\lambda}\right]-s(q)\right]
$$

Here

$$
\begin{aligned}
F_{\lambda}= & \sum_{\imath=0}^{4} \lambda_{i} F^{\imath}=\lambda_{0} \sum_{\alpha} u\left(x_{\alpha}\right)+\sum_{i=1}^{3} \lambda_{i} \sum_{\alpha} u\left(x_{\alpha}\right) p_{\alpha}^{i} \\
& -\lambda_{4} \sum_{\alpha}\left[\phi\left(p_{\alpha}\right)+\frac{1}{2} \sum_{\beta \neq \alpha} V\left(x_{\alpha}-x_{\beta}\right)\right] u\left(x_{\alpha}\right) .
\end{aligned}
$$

We can fix $E^{Q}\left(F^{\imath}\right)=q_{i}$ for $i=0,1, \ldots, 4$ and define the thermodynamic entropy function

$$
\hat{s}(q)=\inf _{Q: E^{Q}\left[F_{2}\right]=q_{i}} s(Q) .
$$

Then $\hat{s}(q)$ is a convex function of $q \in \mathbb{R}^{5}$ and $\psi$ and $\hat{s}$ are related by

$$
\psi(\lambda)=\sup _{q}\left[\sum_{i=0}^{4} \lambda_{\imath} q_{\imath}-\hat{s}(q)\right]
$$

There is an open subset of $U$ of $L_{0} \times \mathbb{R}$ and an open subset $\hat{U}$ of $\mathbb{R}^{5}$ such that $\nabla \psi$ and $\nabla \hat{s}$ map each onto the other in a smooth one-to-one manner (cf. [R]). Moreover by restricting to a smaller nonempty subset we can assume that the variation in (5.10) 
is attained at a unique measure $Q_{\lambda}$ and $Q_{\lambda}$ is the "Gibbs" measure corresponding to the activity $\lambda$. From the uniqueness it follows that $Q_{\lambda}$ is ergodic as a stationary point process.

Now we want to study local "Gibbs" families of measures. Let $\lambda(x)$ be a smooth map of $T^{3} \times U$. Let $q(x)$ be the corresponding map into $\hat{U}$. Assume $\int_{T^{3}} q_{0}(x) d x=1$.
Let us define on $\left(T^{3} \times \mathbb{R}^{3}\right)^{N}$

$$
G(\underline{x}, \underline{p})=\sum_{\alpha} \lambda_{0}\left(x_{\alpha}\right)+\sum_{\alpha} \sum_{i=1}^{3} \lambda_{i}\left(x_{\alpha}\right) p_{\alpha}^{i}-\sum_{\alpha} \lambda_{4}\left(x_{\alpha}\right) h_{\alpha}
$$

where

$$
h_{\alpha}=\phi\left(p_{\alpha}\right)+\frac{1}{2} \sum_{\beta \neq \alpha} V\left(\frac{x_{\alpha}-x_{\beta}}{\varepsilon}\right)
$$

and $N \rightarrow \infty$ as $\varepsilon \rightarrow 0$ with $N \varepsilon^{3} \rightarrow 1$.

We define on $\left(T^{3} \times \mathbb{R}^{3}\right)^{N}$ a local Gibbs measure $\hat{\mu}_{N}=\hat{g}_{N} d \underline{x} d \underline{p}$ with density given by

$$
\hat{g}_{N}(\underline{x}, \underline{p})=\frac{1}{Z_{N}^{c}} \exp [G(\underline{x}, \underline{p})] .
$$

Note that $\hat{g}_{N}$ is the same function as defined in 2.27. According to Theorem 5.6

$$
\begin{aligned}
\lim _{\varepsilon \rightarrow 0} \frac{1}{N} \log Z_{N}^{c} & =\sup \left\{\int_{T^{3}} d x\left[E^{Q x}\left[F_{x}(\omega)\right]-s\left(Q_{x}\right)\right] ;\left\{Q_{x}\right\}: \int \varrho\left(Q_{x}\right) d x=1\right\} \\
& =\sup _{\zeta: \int \zeta_{0}(x) d x=1}\left\{\int\left[\sum_{i} \lambda_{\imath}(x) \zeta_{i}(x)-\hat{s}(\zeta(x))\right] d x\right\} .
\end{aligned}
$$

Since the supremum is attained anyway at a $\zeta_{0}(x)$ with $\int \zeta_{0}(d x)=1$, we can drop the restriction and

$$
\lim _{N \rightarrow \infty} \frac{1}{N} \log Z_{N, \lambda}^{c}=\int \psi(\lambda(x)) d x .
$$

Let us take a function $\left[h_{\alpha}\right.$ defined in (5.14)]

$$
\begin{aligned}
\Omega(\underline{x}, \underline{p})= & N^{-1}\left\{b_{0}(x)+\sum_{i=1}^{3} \sum_{\alpha} b_{i}\left(x_{\alpha}\right) p_{\alpha}^{i}+\sum_{\alpha} b_{4}\left(x_{\alpha}\right) h_{\alpha}\right\} \\
& +a \int K\left(x, \omega_{x, \varepsilon}\right) d x,
\end{aligned}
$$

where $b_{i}(x)$ are continuous, $b_{4}<\lambda_{4}$ and $K(x, \omega)$ is bounded and local. Then if we define the partition function

$$
\bar{Z}_{N}=\int \exp [\Omega(\underline{x}, \underline{p})] d \hat{\mu}_{N},
$$

then

$$
\begin{aligned}
\lim _{N \rightarrow \infty} \frac{1}{N} \log \bar{Z}_{N}= & \sup _{\left\{Q_{x}\right\} ; \int \zeta_{0}(x) d x=1} \int\left[E^{Q x}\left(\Omega_{x}(\omega)\right)+\sum_{i} \lambda_{i}(x) \zeta_{i}(x)-s\left(Q_{x}\right)\right] d x \\
& -\int \psi(\lambda(x)) d x
\end{aligned}
$$


where $\zeta_{i}(x)=E^{Q x}\left[F_{i}\right]$. Clearly one can move the sup inside and drop the constraint $\int \zeta_{0}(x) d x=1$ to have an upper bound. Together with (5.11) we have

$$
\begin{aligned}
& \lim _{N \rightarrow \infty} N^{-1} \log \bar{Z}_{N} \\
& \quad \leq \int \sup _{Q}\left\{E^{Q}\left(K\left(x, \omega_{x, \varepsilon}\right)\right)+\sum_{\mu} b_{\mu}(x) \zeta_{\mu}-I(\zeta, \lambda(x))\right\} d x .
\end{aligned}
$$

Here

$$
I(\zeta, \lambda)=\hat{s}(\zeta)+\psi(\lambda)-\lambda \zeta
$$

These estimates provide an easy corollary.

Corollary 5.8. The measures $\hat{\mu}_{N}$ have a law of large numbers. If

$$
m=\int E^{\hat{\mu}_{\lambda(x)}}\left(\Omega_{x}(\omega)\right) d x,
$$

where $\hat{\mu}_{\lambda(x)}$ is the Gibbs point process corresponding to $\lambda(x)$ then

$$
\hat{\mu}_{N}\left\{(\underline{x}, \underline{p}):\left|\frac{1}{N} \Omega(\underline{x}, \underline{p})-m\right| \geq \delta\right\}_{N \rightarrow \infty} \rightarrow 0
$$

exponentially fast in $N$ for each $\delta>0$.

Let us map the configuration $(\underline{x}, \underline{p})$ into the set of maps from $T^{3}$ into the stationary point processes on $\mathbb{R}^{3} \times \mathbb{R}^{3}$. Let $K$ be an arbitrary number. We map the configuration in a box of size $\varepsilon K$ around $x \in T^{3}$ to a configuration in a box of size $K$ in $\mathbb{R}^{3}$ by $y_{\alpha}=\left(x_{\alpha}-x\right) \varepsilon^{-1}$. Then $\left\{\left(y_{\alpha}, p_{\alpha}\right)\right\}=\omega_{k}^{x}$ is a configuration in $B_{k} \times \mathbb{R}^{3}$. We can extend it periodically to a configuration $\tilde{\omega}_{k}^{x}$ in $\mathbb{R}^{3} \times \mathbb{R}^{3}$, and averaging over translations on $B_{k}$ we obtain a stationary random point process $R_{x, \varepsilon, k, \omega}$ defined by

$$
E^{R_{x, \varepsilon, k, \omega}}\left[A\left(\omega^{\prime}\right)\right]=\frac{1}{\left|B_{k}\right|} \int_{B_{k}} d y A\left(\tau_{y} \tilde{\omega}_{k}^{x}\right) .
$$

Let $\hat{\pi}_{N, k}$ be the measures induced by this map by $d \hat{\mu}_{N}=\hat{g}_{N} d \underline{x} d \underline{p}$ on the space of maps

$$
x \in T^{3} \rightarrow R_{x} \in M_{0} .
$$

Our basic result can be thought of as a large deviation result for $\hat{\pi}_{N, k}$. Then the estimate (5.20) can be rewritten as $\left(\zeta_{\mu}(x)=E^{Q_{x}}\left(F_{\mu}\right)\right)$

$$
\begin{aligned}
\lim _{k \rightarrow \infty} & \lim _{\varepsilon \downarrow 0} \frac{1}{N} \log E^{\hat{\pi}_{N, k}}\left(\exp N \int_{T^{3}} d x E^{R_{x, w}}\left(\Omega_{x}\right)\right) \\
= & \sup _{\left\{Q_{x}\right\}: \int \zeta_{0}(x) d x=1} \int d x\left[E^{Q_{x}}\left(\Omega_{x}\right)+\sum_{\mu} \lambda_{\mu}(x) \zeta_{\mu}(x)\right. \\
& \left.-s\left(Q_{x}\right)-\psi(\lambda(x))\right]
\end{aligned}
$$


Proof of Lemma 3.8. By entropy bound (2.28) the expectation with respect to $g_{N}$ is bounded by

$$
N^{-1} \delta_{0}^{-1} \log E^{\hat{g}_{N}}\left[\exp \left\{\delta_{0} N \int_{T^{3}}\left(1-\sigma_{k}\right) A d x\right\}\right]+\delta^{-1} N^{-1} H .
$$

The first term can be bounded using (5.21) in the limit $N \rightarrow \infty$ and $k \rightarrow \infty$ by

$$
\delta_{0}^{-1} \int \sup _{\zeta(x)}\left\{\delta_{0} \sum_{\mu} b_{\mu}(x) \zeta_{\mu}(x)\left(1-1_{u_{2}}(\zeta(x))-I(\zeta(x), \lambda(x))\right\} d x\right.
$$

Since $I(\zeta(x), \lambda(x))>\varepsilon>0$ in the region $1-1_{u_{2}}(\zeta(x)) \neq 0$ and $I$ is convex in $\zeta(x)$, the sup in (5.23) is zero if $\delta_{0}$ is small enough. We have thus proved Lemma 3.8.

We can now prove Lemma 4.2.

Proof of Lemma 4.2. Let $Q$ be any limit of $\bar{Q}_{\varepsilon}$. By (5.9)

$$
h\left(Q \mid P_{\delta}\right) \leq E^{Q}\left(F_{\lambda, \delta}\right)-\psi(\lambda) .
$$

By the entropy inequality and 4.4

$$
E^{Q}\left(F_{\lambda, \delta}\right) \leq \lim _{N \rightarrow \infty} \frac{1}{N} \log E^{P_{N}}\left(e^{N \int F_{\lambda, \delta}\left(\omega_{\varepsilon, x}\right) d x}\right)+C \leq \psi(\lambda)+C
$$

where the last inequality is due to 5.17 .

Finally we state:

Lemma 5.9. If $g_{N}(\underline{x}, \underline{p})$ is a symmetric probability density on $\left(T^{3} \times \mathbb{R}^{3}\right)^{N}$ such that the relative entropy with respect to $\hat{g}_{N, \lambda}$

$$
H\left(g_{N} \mid \hat{g}_{N, \lambda}\right) \leq c N
$$

Then

$$
E^{g_{N}}\left(\frac{1}{N} \sum_{(\alpha \neq \beta)} V\left(\frac{x_{\alpha}-x_{\beta}}{\varepsilon}\right)\right) \leq c^{\prime}
$$

and therefore by Lemma 5.7

$$
E^{g_{N}}\left(\frac{1}{N} \sum_{(\alpha \neq \beta)} W\left(\frac{x_{\alpha}-x_{\beta}}{\varepsilon}\right)\right) \leq C_{W}
$$

for all $W$ continuous and having compact support.

Proof. It is an easy consequence of the entropy inequality and the theory developed above. 


\section{References}

[CLY] Conlon, J.G., Lieb, E.H., Yau, H.T.: The Coulomb gas at low temperature and low density. Commun. Math. Phys. 125, 153-218 (1989)

[DeM] DeMasi, A., Ianiro, N., Pellegrinotti, A., Presutti, E.: A survey of the hydrodynamical behavior of many-particle systems. In: Nonequilibrium phenomena. II. From stochastic to hydrodynamics. Lebowitz, J.L., Montroll, E.W. (eds.), pp. 123-234, Amsterdam: NorthHolland, 1984

[Sp] Spohn, H.: Large Dynamics of interacting particles. Berlin, Heidelberg, New York: Springer 1991

[Si] Sinai, Ya.G.: Dynamics of local equilibrium Gibbs distributions and Euler equations. The one-dimensional case. Selecta Math. Sov. 7(3), 279-289 (1988)

[BDS] Boldrighini, C., Dobrushin, R.L., Suhov, Yu.M.: One-dimensional hard rod caricatures of hydrodynamics. J. Stat. Phys. 31, 577-616 (1983)

[Y] Yau, H.T.: Relative entropy and the hydrodynamics of Ginzburg-Landau models. Lett. Math. Phys. 22, 63-80 (1991)

[GPV] Guo, M.Z., Papanicolaou, G.C., Varadhan, S.R.S.: Nonlinear diffusion limit for a system with nearest neighbor interactions. Commun. Math. Phys. 118, 31-53 (1988)

[V] Varadhan, S.R.S.: Scaling limits for interacting diffusions. Commun. Math. Phys. 135, 313353 (1991)

[O] Olla, S.: Large deviations for Gibbs random fields. Prob. Theory Related Fields 77, 343-357 (1988)

[OV] Olla, S., Varadhan, S.R.S.: Scaling limit for interacting Ornstein-Uhlenbeck processes. Commun. Math. Phys. 135, 355-378 (1991)

[Re] Rezakhanlou, F.: Hydrodynamic limit for a system with finite range interactions. Commun. Math. Phys. 129, 445-480 (1990)

[R] Ruelle, D.: Statistical Mechanics. Reading, MA: Benjamin 1969

[V1] Varadhan, S.R.S.: Large deviation and applications. Philadelphia: SIAM 1984

Communicated by J.L. Lebowitz 